\title{
An Influence Analytical Model of Dedicated Bus Lane on Network Traffic by Macroscopic Fundamental Diagram
}

\author{
Yingying Ma $(\mathbb{D}$, Yuanqi Xie $\mathbb{D}$, and Yongjie Lin $(\mathbb{D}$ \\ Department of Transportation Engineering, South China University of Technology, Guangzhou 510640, China \\ Correspondence should be addressed to Yongjie Lin; linyjscut@scut.edu.cn
}

Received 30 May 2021; Revised 26 July 2021; Accepted 10 August 2021; Published 24 August 2021

Academic Editor: Ming Xu

Copyright (C) 2021 Yingying Ma et al. This is an open access article distributed under the Creative Commons Attribution License, which permits unrestricted use, distribution, and reproduction in any medium, provided the original work is properly cited.

\begin{abstract}
To study the influence mechanism of dedicated bus lanes on the urban road network, this paper proposes a novel analytical model of macroscopic fundamental diagram (MFD) and passenger macroscopic fundamental diagram (p-MFD) and the corresponding indicators based on MFD and p-MFD to evaluate the operation of the network. Taking the grid network as an example, this paper collects traffic flow to calibrate the developed MFD and p-MFD and evaluates the network performance under different proportions of dedicated bus lanes. The simulation results show that the larger the proportion of dedicated bus lanes, the greater the impact on the rising section and the stable section of MFD and the descending section and post-stable section of p-MFD. Further analysis for the sensitivity of simulation experiments found that the strategy of setting dedicated bus lanes will improve the efficiency of vehicle and passenger transport when the road network is in a smooth state and ensure the continuous output of passengers when the network is in a congested state.
\end{abstract}

\section{Introduction}

The increasing number of vehicles causes the traffic demand for the road network in cities to increase rapidly, and corresponding traffic congestion in many cities is serious. The transit priority strategy of setting dedicated bus lanes plays an important role in improving bus operating efficiency and the level of service. The dedicated bus lane is an economical implementation method to improve the level of public transport service and ease the pressure of urban traffic [1]. A large number of cities in the world have implemented dedicated bus lanes to promote public transport. By the end of 2019, the total length of dedicated bus lanes in China was $14,951.7 \mathrm{~km}$ [2]. The planning and implementation of dedicated bus lanes aim to optimize the allocation of road resources in urban cities and improve traffic conditions for bus priority $[3,4]$.

The implementation of dedicated bus lanes has a certain degree of impact on road network traffic. A comprehensive evaluation of dedicated bus lanes is a necessary step to analyse the feasibility and benefits of its implementation that concerns the trade-off of the benefits of public and private transport in the urban road network. Urban transport systems should meet the travel needs of urban residents. Therefore, when setting up dedicated bus lanes, the impact on the vehicle transport and passenger transport of urban road networks should be comprehensively considered. Moreover, the benefits of dedicated bus lanes are related to the traffic state of the urban road network. In order to determine the effect of setting up dedicated bus lanes, it is important to propose a group of indicators to analyse the overall performance of the road network, which are helpful capture the impact of dedicated bus lanes on the urban road network and understand how it will change with the varying traffic status.

In recent years, the latest trend in the study of urban road network transport is to use the macroscopic fundamental diagram (MFD) to model and analyse urban-scale road network traffic. Daganzo proposed the concept and basic description of the MFD of the urban network in 2007 and verified the existence of MFD using traffic simulation [5] and empirical data [6]. Through the analysis of MFD, Daganzo [7] found that MFD is an inherent attribute of the road network itself, and it remains unchanged when traffic 
demand changes. The basic curve of MFD is a parabola. However, in the actual analysis, it is often simplified to a triangle or trapezoid [8]. MFD not only reflects the general relationship between network traffic flow and network operation level but also describes the relationship between the number of vehicles in the road network and the output flow of the road network [9]. However, the MFD can only express the performance of vehicles in the transport system but not consider the average number of passengers of each transport mode.

In order to improve the concept of MFD, Zheng [10] and Nicolas [11] proposed the concept of passenger macroscopic fundamental diagram ( $\mathrm{p}-\mathrm{MFD}$ ) to evaluate the passenger transport capacity of the multimodal urban network with cars and buses. The existence of MFD [9, 12-15] and p-MFD [16] has been verified by the empirical data of urban road networks around the world. As the inherent attributes of the road network, MFD and p-MFD, respectively, indicate the relationship between the density of vehicles in the road network and the output flow of vehicles of the road network, and also the relationship between the density of vehicles in the road network and the output flow of passengers of the road network. They help to evaluate the performance of the urban multimodal network. In recent years, MFD has been extensively studied and applied, including model formulation $[17,18]$, optimization [19-22], traffic control [23, 24], and perimeter control $[25,26]$.

Most previous studies of the impact of dedicated bus lanes on MFD and p-MFD mainly focus on data sources, modelling methods, analysis factors, and the impact on road network transport. In terms of data sources, MFD can be obtained through traffic software simulation [27-30], theoretical model $[3,10,31]$, and empirical data $[12,16]$, and $\mathrm{p}$-MFD can be derived by fixed passenger occupancy rate [30], estimating passenger occupancy rate $[3,11,12,16,28]$ or using theoretical models [32] on the passenger occupancy rate of each transport mode in the multimodal urban road network. Most previous studies of the impact of dedicated bus lanes on MFD and p-MFD focus on theoretical road networks $[3,10,11,31]$ or actual road networks [12, 16, 27-30,32]. In terms of modelling methods, the existing modelling methods of MFD and p-MFD include scattering plot method [10, 12, 16, 28-30], contour map (3D-MFD) $[12,16,19,20]$, measured or simulated data fitting method $[16,27,28]$, and analytical model method $[3,11,32]$. The literature review on the impact of bus lanes on MFD and p-MFD is summarized in Table 1.

Although existing literature has found that dedicated bus lanes have a certain impact on the road network MFD and $\mathrm{p}-\mathrm{MFD}$, they pay more attention to the changes in shape and the maximum value of MFD and p-MFD under different conditions. Limited attention is paid to propose comprehensively a group of indicators for urban road networks based on MFD and p-MFD and use them as the basis to study the influence of dedicated bus lanes on the operation of the road network under different conditions of the road network, regarding vehicles and passengers, respectively.
Microcosmic traffic evaluation indicators only focus on traffic operations of a certain road section or under a certain network state. Through in-depth study of MFD and p-MFD, the road network can be viewed as a whole, and the traffic state under different conditions can be evaluated. Comparing microcosmic traffic evaluation indicators, MFDbased indicators can more fully reflect the transport efficiency, capacity, and reliability of the road network under different conditions at the network level.

Therefore, this paper proposes a novel description and modelling methods of MFD and p-MFD based on the Gaussian mixture model (GMM). The indicators based on MFD and p-MFD are proposed to analyse comprehensively the operation of the road network. This methodology is suitable for various urban networks, which can be extended to multiple modes (cars, buses, etc.) to evaluate the efficiency and other aspects of the urban network from vehicles and passengers' perspectives. This paper takes the grid network as an example and uses traffic simulation to obtain the traffic data when the proportion of dedicated bus lanes is changed. The characteristic parameters of MFD and p-MFD are analysed in detail to study and summarize the impact mechanism of dedicated bus lanes on the urban network and to support and expand existing research on the impact of dedicated bus lanes on MFD and p-MFD.

\section{Analytical Framework Based on MFD and p-MFD}

2.1. Description of Macroscopic Fundamental Diagram. MFD (macroscopic fundamental diagram) is composed of a series of scattered points, which are from the empirical or experimental data at a certain time interval $\Delta t$ during the operation process of the urban road network. In this paper, MFD takes the vehicle density in the road network $K$ (veh/ $\mathrm{km} / \mathrm{ln}$ ) as the abscissa and the vehicle flow leaving the road network $Q(\mathrm{veh} / \mathrm{ln} / \mathrm{h})$ as the ordinate. According to the definition of MFD, the relevant parameter calculation equation is as follows:

$$
\begin{aligned}
q(t) & =q(t-\Delta t)+q^{i}(t)-q^{o}(t), \\
Q(t) & =\frac{q^{o}(t) \cdot 3600}{\Delta t \cdot N}, \\
K(t) & =\frac{q(t)}{L},
\end{aligned}
$$

where $\Delta t$ represents the time interval for data collection (s), $q(t)$ denotes the number of vehicles running in the road network at time $t$ (veh), $q^{i}(t)$ is the number of vehicles entering the road network at time $t$ (veh), $q^{o}(t)$ represents the number of vehicles leaving the road network at time $t$ (veh), $Q(t)$ means the flow of vehicles leaving the road network at time $t(\mathrm{veh} / \mathrm{ln} / \mathrm{h}), N$ represents the total number of exit lanes of the road network (ln), $K(t)$ represents the density of running vehicles in the road network at time $t$ (veh/ $/ \mathrm{km} / \mathrm{ln}$ ), and $L$ denotes the total length of lanes in the road network $(\mathrm{km})$. 
TABLE 1: Literature review on implementation of dedicated bus lanes on MFD and p-MFD.

\begin{tabular}{|c|c|c|}
\hline & MFD & p-MFD \\
\hline Data sources & $\begin{array}{c}\text { Traffic software simulation }[27-30] \\
\text { Theoretical model }[3,10,31] \\
\text { Empirical data }[12,16]\end{array}$ & $\begin{array}{c}\text { Fixed passenger occupancy rate }[30] \\
\text { Estimated passenger occupancy rate }[3,11,12,16,28] \\
\text { Theoretical models }[32]\end{array}$ \\
\hline Road network & $\begin{array}{l}\text { Theoretical road network }[3,10,31] \\
\text { Actual road network }[12,16,27-30]\end{array}$ & $\begin{array}{c}\text { Theoretical road network }[3,11] \\
\text { Actual road network }[12,16,28,30,32]\end{array}$ \\
\hline Modelling method & $\begin{array}{l}\text { Scatter plot }[10,12,29] \text { and contour map (3D-MFD) } \\
{[12,29]} \\
\text { Empirical or simulated data fitting: least square method } \\
\text { and exponential function fitting (3D-MFD) }[16,27,28] \\
\text { Analytical model: variational method }[3]\end{array}$ & $\begin{array}{l}\text { Scatter plot }[12,28,30] \text { and contour map (3D-MFD) } \\
{[12,16,30]} \\
\text { Empirical or simulated data fitting: least square method and } \\
\text { exponential function fitting (3D-MFD) [16] } \\
\text { Analytical model: variational method }[3,32] \text {, the analytical } \\
\text { model considering the proportion of passengers choosing car } \\
\text { transport and public transport }[11]\end{array}$ \\
\hline Analysis factors & The shape of MFD (maximum) $[3,10,16,27-29]$ & The shape of p-MFD (maximum) $[3,11,12,16,28,30,32]$ \\
\hline $\begin{array}{l}\text { Impact on road } \\
\text { network transport }\end{array}$ & $\begin{array}{l}\text { (i) The shape of the MFD of the dedicated bus lane is } \\
\text { similar to the shape of the MFD of the mixed traffic } \\
\text { [27,28], and its shape is related to the average bus dwell } \\
\text { time [28] and headway [3]. } \\
\text { (ii) The number of vehicles in the road network when } \\
\text { setting up dedicated bus lanes will not reach the } \\
\text { maximum value of the MFD of the previous road } \\
\text { network [10,11,29]. } \\
\text { (iii) With a very low accumulation of buses, the road } \\
\text { network achieves the best operating conditions } \\
\text { (maximum capacity value). When the proportion of } \\
\text { dedicated bus lanes increases, the transport } \\
\text { performance of cars gets worse and the transport } \\
\text { performance of buses improves [29]. } \\
\text { (iv) When dedicated bus lanes are available, buses have } \\
\text { significantly improved speed and flow compared with } \\
\text { mixed flow conditions [16]. } \\
\text { (v) In the case of car traffic congestion, increasing the } \\
\text { space of dedicated bus lanes can reduce the total cost of } \\
\text { society and increase the share rate of buses [31]. }\end{array}$ & $\begin{array}{l}\text { (i) The number of passengers in the road network when } \\
\text { setting up dedicated bus lanes will not reach the maximum } \\
\text { value of the p-MFD of the previous road network [3]. } \\
\text { (ii) To obtain more passenger throughput, when the road } \\
\text { network has implemented dedicated bus lanes, more buses } \\
\text { can be deployed in the network [28]. A certain number of } \\
\text { buses can improve the transport performance of the road } \\
\text { network [30]. } \\
\text { (iii) The increase in the proportion of bus lanes will reduce } \\
\text { the marginal effect of the speed of the bus fleet [12]. } \\
\text { (iv) Under noncongested traffic conditions, mixed lanes can } \\
\text { always produce higher passenger throughput. However, as } \\
\text { the degree of congestion increases, the passenger throughput } \\
\text { of dedicated bus lanes is greater than that of mixed lanes [32]. } \\
\text { (v) The dedicated bus lane ensures that the bus system can } \\
\text { still operate even when the road network is very crowded } \\
\text { [11]. }\end{array}$ \\
\hline
\end{tabular}

Using network traffic empirical or experimental data, according to equations (1)-(3), a scatter diagram of MFD can be obtained as shown in Figure 1. In-depth research found that in the complete road network MFD, as the density of running vehicles in the road network $K$ increases from 0 , the flow of vehicles leaving the road network $Q$ has a significant upward, stable, and downward trend. Then, $Q$ drops to the minimum value $Q_{\min }$ since the downtrend occurred. As $K$ continues to increase, $Q$ maintains fluctuations within a certain range above and below $Q_{\min }$. If $Q_{\min }$ is obviously greater than 0 at this time, it means that there are still a small number of vehicles in the road network that can leave the road network, such as the vehicles near the exit of the road network. If $Q_{\min }$ is close to 0 at this time, it means that the road network has a deadlock and the majority of vehicles on the road network cannot leave the road network.

\subsection{Description of Passenger Macroscopic Fundamental} Diagram. The concept of the p-MFD is proposed to evaluate the passenger transport capacity of the multimodal road network in this study. Cars can travel according to the demand of car users when the road network condition permits. As the public transport mode, the bus must travel along the preset bus lanes, and the departure interval has certain restrictions. The bus is an independent mode of transport, which is different from the car in terms of vehicle structure, passenger capacity, and driving characteristics. Therefore, there are much difference in operation characteristics between cars and buses. As the road network traffic facilities or traffic management measures (bus lanes, bus priority, etc.) change, the differences between the two are significant. Car p-MFD and bus p-MFD will also change accordingly, and there may be differences in the changing patterns. Therefore, this paper studies the bus p-MFD and car p-MFD of urban road networks separately to study better the changes in the capacity of these two modes to transport passengers when the states of the road network change.

p-MFD is composed of a series of scattered points. The scattered points are the empirical or experimental data at a certain time interval $\Delta t$ during the operation process of the road network. In this paper, p-MFD takes the vehicle density in the road network of $K(\mathrm{veh} / \mathrm{km} / \mathrm{ln})$ as the abscissa and the passenger flow leaving the road network of PAX (person $/ \mathrm{ln} / \mathrm{h}$ ) as the ordinate. According to the definition of $\mathrm{p}-\mathrm{MFD}$, the relevant parameter calculation equation is as follows: 


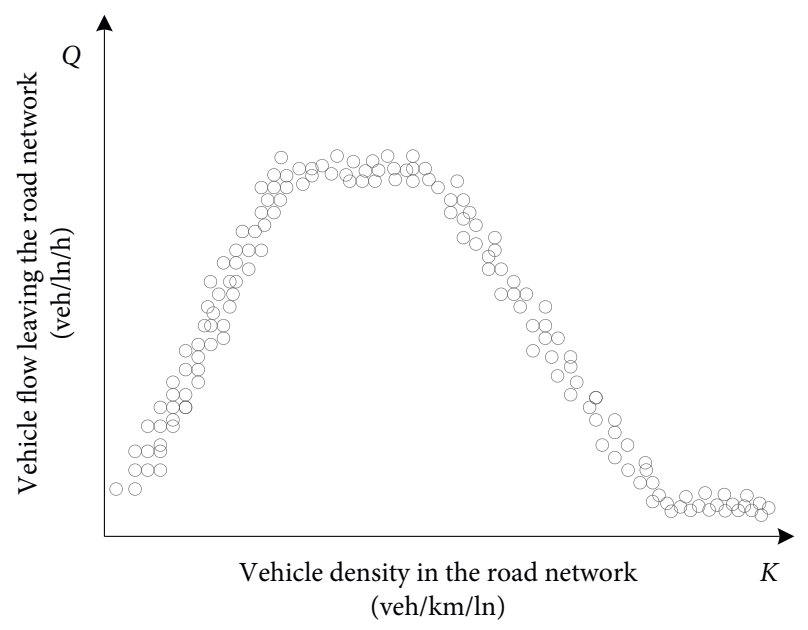

FIgURE 1: MFD.

$$
\begin{aligned}
P_{c}(t) & =\frac{n_{c} \cdot\left[q_{c}(t-\Delta t)+q_{c}^{i}(t)-q_{c}^{o}(t)\right] \cdot 3600}{\Delta t \cdot N}, \\
P_{b}(t) & =\frac{n_{b} \cdot\left[q_{b}(t-\Delta t)+q_{b}^{i}(t)-q_{b}^{o}(t)\right] \cdot 3600}{\Delta t \cdot N}, \\
P(t) & =P_{c}(t)+P_{b}(t) \\
K(t) & =\frac{q(t)}{L}
\end{aligned}
$$

where $\Delta t$ represents the time interval for data collection (s), $q(t)$ means the number of vehicles running in the road network at time $t$ (veh), $q_{c}^{i}(t)$ represents the number of cars entering the road network at time $t$ (veh), $q_{b}^{i}(t)$ represents the number of buses entering the road network at time $t$ (veh), $q_{c}^{o}(t)$ represents the number of cars leaving the road network at time $t$ (veh), $q_{b}^{o}(t)$ denotes the number of buses leaving the road network at time $t$ (veh), $n_{c}$ represents the average passenger occupancy of cars (person), $n_{b}$ represents the average passenger occupancy of buses (person), $P_{c}(t)$ means the flow of car passengers leaving the road network at time $t$ (person/ln/h), $P_{b}(t)$ represents the flow of bus passengers leaving the road network at time $t$ (person/ln/h), $N$ represents the total number of exit lanes of the road network $(\ln ), K(t)$ represents the density of running vehicles in the road network at time $t(\mathrm{veh} / \mathrm{km} / \mathrm{ln})$, and $L$ represents the total length of lanes in the road network $(\mathrm{km})$.

2.3. Segmented Linear Model of MFD and p-MFD. The MFD scatter plots of different road networks, car p-MFD, and bus p-MFD have similar changing trends, namely the rising, stable, descending, and post-stable trends. The post-stable trend with consistently low value means that a very small number of vehicles still can leave the road network even when the road network is seriously congested, such as the vehicles near the exit. However, there are still some differences between MFD and p-MFD. Therefore, the scattered points of MFD and p-MFD can be clustered into several categories according to the changing trend. Based on research [33], this paper uses Gaussian mixture clustering to classify the scattered points of MFD and p-MFD. The Gaussian mixture model (GMM) is a linear combination of multiple Gaussian models and is a common clustering method. This method uses the Gaussian probability density function to calculate the probability that the scatter points belong to each category. The category with the highest probability of the scatter points is regarded as the category to which the scatter points belong, and the scatter points are aggregated into several categories with obvious differences. GMM can divide the data with any shape of an ellipse and has a better clustering effect on curved data points. The sklearn.mixture library is used in the open-source software of python to implement GMM in this paper.

The probability distribution of the Gaussian mixture model is shown in the following equation:

$$
P(x \mid \theta)=\sum_{m=1}^{M} \alpha_{m} \varphi\left(x \mid \theta_{m}\right),
$$

where $M$ represents the number of sub-Gaussian models in the mixture model, $m=1,2, \ldots, M, M \geq 3 ; \alpha_{m}$ means the probability that the scatter points belong to the $m$ th submodel, $a_{m} \geq 0, \sum_{m=1}^{M} a_{m}=1$; and $\varphi\left(x \mid \theta_{m}\right)$ represents the Gaussian distribution density function of the $m^{\text {th }}$ sub-model, $\theta_{m}=\left(\mu_{m}, \sigma_{m}^{2}\right)$.

According to equations (1)-(3) and (7)-(10), MFD and p-MFD scatter plots of various modes can be obtained. The GMM model is used to cluster the MFD and the p-MFD scattered points of cars and buses separately and divide them into $M$ categories. The $M$-segment scatter points of the road network MFD, the car p-MFD, and the bus p-MFD are linearly fitted and represented by a segmented linear model as follows:

$$
\begin{aligned}
& F_{w}(k)= \begin{cases}a_{w}^{1} k+b_{w}^{1}, & 0 \leq k \leq k_{w}^{1}, \\
a_{w}^{2} k+b_{w}^{2}, & k_{w}^{1}<k \leq k_{w}^{2}, \\
\cdots & \\
a_{w}^{M-1} k+b_{w}^{M-1}, & k_{w}^{M-2}<k \leq k_{w}^{M-1}, \\
a_{w}^{M} k+b_{w}^{M}, & k_{w}^{M-1}<k,\end{cases} \\
& k_{w}^{j}=\frac{b_{w}^{j}-b_{w}^{j-1}}{a_{w}^{j-1}-a_{w}^{j},} \quad j=2, \ldots, M,
\end{aligned}
$$

where $F=\{Q, P A X\}$. When $F=Q, Q_{w}(k)$ represents the flow of vehicles leaving the road network when the density is $k(\mathrm{veh} / \mathrm{ln} / \mathrm{h}) . w=\{$ net $\}$, net represents the road network. When $F=\operatorname{PAX}, \operatorname{PAX}_{w}(k)$ represents the passenger flow of cars or buses leaving the road network when the density is $k$ (person $/ \mathrm{ln} / \mathrm{h}$ ). $w=\{c, b$, net $\}$, where $c$ represents cars, $b$ is buses, and net means the road network. $F_{w}^{j}(k)=a_{w}^{j} k+b_{w}^{j}$, where $j=1,2, \ldots, M, j$ represents the corresponding number of each segment, $a_{w}^{j}$ denotes the slope of the function, $b_{w}^{j}$ means the constant of the function, and $k_{w}^{j}$ 
represents the corresponding vehicle density $k$ in the road network at the beginning of the $j$ segment of MFD or p-MFD. In this paper, $k_{w}^{1}$ is generally considered to be 0 .

To avoid state mutation in MFD and p-MFD, if $a_{w}^{j} a_{w}^{j+2}<0, j=1,2, \ldots, M-2$, then $a_{w}^{j}=0$. When $a_{w}^{j}>0$, the segment is in a rising state. When $a_{w}^{j}=0$, the segment is in a steady state. When $a_{w}^{j}<0$, the segment is in a descending segment. The rising section consists of multiple consecutive rising segments, and the descending section consists of multiple consecutive descending segments.

\subsection{MFD and p-MFD Modelling}

\subsubsection{MFD Modelling}

Step1: Use empirical or experimental data to calculate the vehicle density $K(t)$ in the road network and the flow of vehicles leaving the road network $Q(t)$ according to equations (1)-(3)

Step2: Use $K(t)$ as the abscissa and $Q(t)$ as the ordinate to obtain the scatter plot of road network MFD

Step3: Employ GMM to cluster road network MFD scatters and divide scatters into $M$ categories

Step4: Linear fitting is performed on the $M$-segment scatters of the road network MFD, and the piecewise linear function is used to represent the road network MFD model

According to the changing trend of the scatter plot of road network MFD, the number of clusters of MFD $M$ is set to 4 in this paper. The 4 -segment scattered points of MFD classified by GMM are linearly fitted, and MFD is represented by a piecewise linear function,. MFD obtained by the above modelling method is shown in Figure 2.

According to Figure 2, each section of MFD can be described as follows:

(1) Rising Section. The traffic in the road network is in a smooth state, and the throughput of cars and buses in the road network is smooth. At this section, $Q(t)$ increases with the increase of $K(t)$.

(2) Stable Section. The traffic in the road network is still in a saturated state, and the road network continues to transport cars and buses. As $K(t)$ increases, $Q(t)$ stabilizes within a certain range, and $Q(t)$ reaches the maximum value $Q_{\max }$. If $K(t)$ continues to increase, $Q(t)$ fluctuates around $Q_{\max }$.

(3) Descending Section. The traffic in the road network is in a front-congested state. Some intersections and road sections in the road network are blocked, and part of vehicles cannot leave the road network due to traffic congestion. At this time, the road network has reached the critical point where it can effectively bear no more vehicles. As $K(t)$ continues to increase, $Q(t)$ continues to decrease.

(4) Post-Stable Section. The traffic in the road network is in a post-congested state, and most intersections and road sections in the road network remain seriously blocked, and the corresponding most vehicles cannot leave the road network. $Q(t)$ is stable within a certain range, and $Q(t)$

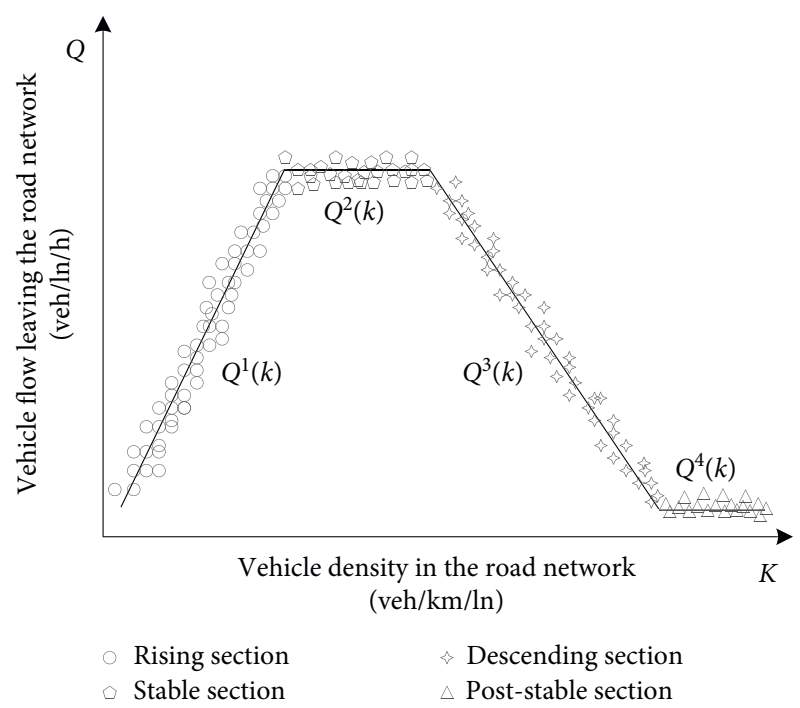

FIGURE 2: MFD obtained by the proposed method.

reaches the minimum value $Q_{\min }$ since MFD enters the descending stage. If $K(t)$ continues to increase, $Q(t)$ will fluctuate around $Q_{\min }$

\subsection{2. $p$-MFD Modelling}

Step1: Use empirical or experimental data to calculate the vehicle density $K(t)$ in the road network, the number of passenger-car users leaving the road network $P_{c}(t)$ and bus passengers leaving the road network $P_{b}(t)$ according to equations (4)-(7)

Step2: Use $K(t)$ as the abscissa and $P_{c}(t)$ and $P_{b}(t)$ as the ordinate separately to obtain the scatter plot of car p-MFD and bus p-MFD, respectively

Step3: Use GMM to cluster the car p-MFD and the bus p-MFD scatters and divide scatters into $M$ categories separately

Step4: Linear fitting is performed on the $M$-segment scatters of car p-MFD and bus p-MFD, and the piecewise linear function is used to represent car p-MFD and bus p-MFD model

Step5: Add the piecewise linear function of car p-MFD and bus p-MFD to get road network p-MFD model, namely

$$
\begin{aligned}
\operatorname{PAX}(k) & =\operatorname{PAX}_{c}(k)+\operatorname{PAX}_{b}(k), \\
g_{p}(k) & =g_{p c}(k)+g_{p b}(k),
\end{aligned}
$$

where $\operatorname{PAX}(k)$ denotes the passenger volumes leaving the road network when the density is $k$ (persons $/ \mathrm{ln} / \mathrm{h}$ ), $\operatorname{PAX}_{c}(k)$ denotes the flow of car passengers leaving the road network when the density is $k$ (persons $/ \mathrm{ln} / \mathrm{h}$ ), $\operatorname{PAX}_{b}(k)$ represents the flow of bus passengers leaving the road network when the density is $k$ (persons $/ \mathrm{ln} / \mathrm{h}), g_{p b}(k)$ denotes the slope of road network p-MFD when the density is $k, g_{p c}(k)$ means the slope of car p-MFD when the density is 
$k$, and $g_{p b}(k)$ denotes the slope of bus p-MFD when the density is $k$.

According to the changing trend of $\mathrm{p}-\mathrm{MFD}$, the number of p-MFD scatter clusters for cars and buses in this paper is set to 4 , namely, $M=4$. Therefore, there are 3 inflection points for the p-MFD of the car and the bus, $k_{w}^{i}, w=\{c, b\}$, $i=1,2,3$. Because the operating characteristics of cars and buses are different, the inflection points of car p-MFD and bus p-MFD may also be different. Therefore, the road network p-MFD obtained by equation (12) is a linear function with $s$ inflection points $(3 \leq s \leq 6)$, and $s=M-1$. The p-MFD obtained using the above method is shown in Figure 3.

In Figure 3, the sections of car p-MFD, bus p-MFD, and road network p-MFD are described as follows:

(1) Rising Section. The traffic in the road network is in a smooth state, and the throughput of car users and bus passengers in the road network is smooth. $P_{c}(t), P_{b}(t)$, and $P(t)$ increase with the increase of $K(t)$, respectively. The rising section of $P(t)$ may have multiple segments depending on the slope.

(2) Stable Section. The traffic in the road network is in a saturated state, and the road network continues to carry car and bus passengers. As $K(t)$ increases, $P_{c}(t)$ and $P_{b}(t)$ stabilize within a certain range and reach the maximum $P_{c \text { max }}$ and $P_{b \max }$, respectively. As $K(t)$ continues to increase, $P_{c}(t)$ fluctuates around $P_{c \max }$, and $P_{b}(t)$ fluctuates around $P_{b \max }$. Notably, $P_{c}(t)$ and $P_{b}(t)$ do not reach the maximum value at the same time, that is, $k_{c}^{1}$ and $k_{b}^{1}$ are not necessarily the same, respectively. When $P_{c}(t)$ and $P_{b}(t)$ enter the stable section, $P(t)$ enters the stable section, too.

(3) Descending Section. The traffic in the road network is in a front-congested state. Some intersections and road sections in the road network are blocked, and some bus and car passengers cannot leave the road network. As $K(t)$ continues to increase, $P_{c}(t), P_{b}(t)$, and $P(t)$ continues to decrease. The descending section of $P(t)$ may have multiple segments depending on the slope.

(4) Post-Stable Section. The traffic in the road network is in a post-congested state, and most of the intersections and road sections are seriously blocked. Without public bus priority measures (dedicated bus lanes, bus signal priority, etc.), most of the car and bus passengers cannot leave the road network. As $K(t)$ increases, $P_{c}(t)$ and $P_{b}(t)$ are stable within a certain range. Then, $P_{c}(t)$ and $P_{b}(t)$ reach the minimum value $P_{c \text { min }}$ and $P_{b \min }$ since car p-MFD and bus p-MFD entered the descending stage separately. As $K(t)$ continues to increase, $P_{c}(t)$ fluctuates around $P_{c m i n}$, and $P_{b}(t)$ fluctuates around $P_{b \min }$. It is worth noting that $P_{c}(t)$ and $P_{b}(t)$ do not reach the minimum value at the same time, namely $k_{c}^{3}$ and $k_{b}^{3}$ are not necessarily the same, respectively. When $P_{c}(t)$ and $P_{b}(t)$ enter the post-stable section, $P(t)$ also enters the post-stable section.

\section{Network Evaluation Based on MFD and $\mathbf{p}$-MFD}

This study proposes several indicators to evaluate the urban road network based on MFD and p-MFD from different

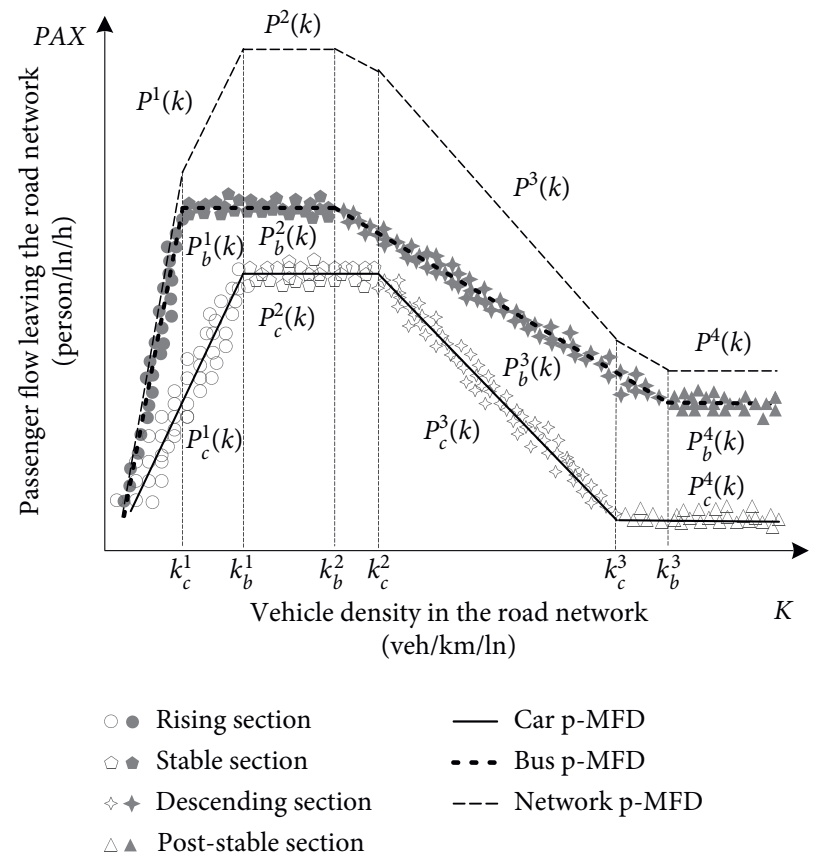

Figure 3: p-MFD obtained by the proposed modelling method.

perspectives. Through the analysis of these indicators, it is helpful analyse more comprehensively the operation of the urban road network under different scenarios.

3.1. Road Network Transport Efficiency. The indicators in this section are proposed to evaluate the operational efficiency of the urban road network based on MFD and p-MFD proposed in the previous section.

The slope of the MFD $g_{c}$ (p-MFD $g_{p}$ ) is defined as the ratio between the increment of traffic flow $\Delta Q$ (passengers $\triangle \mathrm{PAX}$ ) leaving the road network and the increment of vehicle density in the road network $\Delta K$ :

$$
\begin{aligned}
& g_{c}=\frac{\Delta Q}{\Delta K}, \\
& g_{p}=\frac{\Delta \mathrm{PAX}}{\Delta K} .
\end{aligned}
$$

In this study, the slopes of the rising section and the descending section are mainly studied. When the slope of the rising section is larger, the increment in the flow of passengers (vehicles) leaving the road network is larger when the increment in vehicle density is the same. Thus, the efficiency of transporting passengers (vehicles) improves when traffic flow on the road network is in a smooth state; otherwise, the efficiency of transporting passengers (vehicles) deteriorates when the road network is in a smooth state. When the absolute value of the slope of the descending section is larger, the increment of passengers (vehicles) flow leaving the road network is greater when the increment in vehicle density is the same. It reveals that the efficiency of transporting passengers (vehicles) improves when the road network is in a congested state; otherwise, it reveals that the 
efficiency of transporting passengers (vehicles) deteriorates when the road network is in the congested state.

The value of the stable section $F_{w}^{2}(k)$ is defined as the corresponding flow of vehicles leaving the road network in the stable section. In the same way, the value of the poststable section $F_{w}^{4}(k)$ is defined as the corresponding flow of vehicles leaving the road network in the post-stable section as follows:

$$
\begin{aligned}
& F_{w}^{2}(k)=a_{w}^{2} k+b_{w}^{2}, \\
& F_{w}^{4}(k)=a_{w}^{4} k+b_{w}^{4} .
\end{aligned}
$$

In this study, when the stable or post-stable section is close to horizontal, $a_{w}^{2}=0$ or $a_{w}^{4}=0$, and $F_{w}^{2}(k)=b_{w}^{2}$, $F_{w}^{2}(k)=b_{w}^{2}$. Within the density range of the stable section, when the value of the stable section is larger, the flow of passengers (vehicles) leaving the road network is larger, which indicates that the road network is more efficient in transporting passengers (vehicles) in a saturated state. The above indication also applies to the post-stable section.

3.2. Road Network Capacity and Reliability. The indicators proposed in this section are used to evaluate the capacity and reliability of the urban road network based on MFD and p-MFD.

The front-inflection point $\left(k_{1}\right.$ and $\left.q_{\max }\right)$ is defined as the connecting point between the rising and the stable sections, and the back-inflection point $\left(k_{2}, q_{\max }\right)$ is defined as the connecting point between the stable and the descending sections. The schematic diagram is shown in Figure 4.

When the inflection point moves toward the zero point, the abscissa and ordinate values of the inflection point decrease. If the front-inflection point is closer to the zero point, it means that the road network quickly enters the stable state from the smooth one; if the back-inflection point is closer to zero, it shows the road network quickly changes from the stable state to the congested one. Therefore, the faster the road network state changes and the lower the road network throughput is, the less effective the resource utilization of road network is.

The density range of $x$ section $\Delta k_{\text {range }}^{x}$ is defined as the difference of the vehicle density corresponding to the end of the $x$ section minus the vehicle density corresponding to the beginning of the $x$ section. The expression is as follows:

$$
\Delta k_{\text {range }}^{x}=k_{e}^{x}-k_{b}^{x},
$$

where $k_{2}^{x}$ denotes the vehicle density corresponding to the end of the $x$ section, $k_{1}^{x}$ denotes the vehicle density corresponding to the beginning of the $x$ section, $\Delta k_{\text {range }}^{x}$ denotes the density range of $x$ section. $x=\{r, s, d, p\}$, where $r$ represents the rising section, $s$ represents the stable section, $d$ represents the descending section, and $p$ represents the poststable section. When the density range is larger, it means that the road network has a stronger ability to output traffic continuously in this state, and the road network has a stronger continuity in this state.

The goodness of fit, $R^{2}$, is defined as the degree of the regression line fitting the observed values:

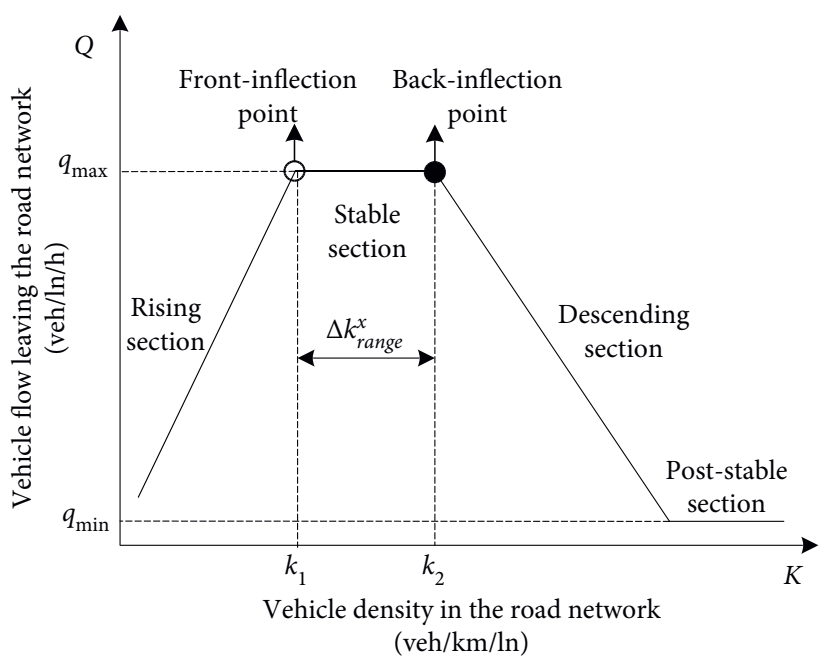

Figure 4: Schematic diagram of indicators.

$$
R^{2}=\frac{\sum_{g=1}^{G}\left(\widehat{y}_{g}-\bar{y}\right)^{2}}{\sum_{g=1}^{G}\left(y_{g}-\bar{y}\right)^{2}},
$$

where $R^{2}$ denotes the goodness of linear fitting; $\widehat{y}_{g}$ denotes the value of the fitted data, which is the data value obtained by linear fitting in this paper; $\bar{y}$ denotes the mean value of the empirical data or experimental data, which is the mean value of the simulated data in this paper; $y_{g}$ denotes the empirical data or experimental data, which is the simulated data in this paper; $G$ denotes the number of data samples; and $g$ denotes the $g^{\text {th }}$ data sample. When the goodness of fit, $R^{2}$, is larger, the scattered points of this section are more concentrated, which means that the stability of the road network transport in this state is better. This study mainly studied the goodness of fit of the rising section and the descending section, respectively.

To sum up, the aforementioned indicators and the symbolic meanings corresponding to the changes in indicators for road network evaluation are summarized in Table 2.

\section{Experimental Design and Results}

4.1. Introduction of the Experiment. Actually, the MFD and p-MFD method and the corresponding indicators proposed in this paper can be extended to multiple modes (cars, buses, etc.) to evaluate the operation of various urban road networks when the traffic improvement is implemented. For example, the implementation of a dedicated bus lane is a very effective measure to improve bus operation. However, it may cause traffic congestion for private cars due to the reduction of road resources. Next, MFD and p-MFD are used to evaluate the influences of dedicated bus lanes on the road network operation in this experiment. A grid road network with signalized intersections is taken for experiments, which is similar to the urban network studied in reference [10]. The roads are divided into main roads, secondary roads, and branch roads. The main roads are 3,000 meters long with six lanes, and each lane is 3.5 
TABLE 2: Road network evaluation indicators based on MFD and p-MFD.

\begin{tabular}{|c|c|c|c|}
\hline Category & Indicator & Trend & Symbolic meaning \\
\hline \multirow{12}{*}{$\begin{array}{l}\text { Road network } \\
\text { transport efficiency }\end{array}$} & \multirow{3}{*}{$\begin{array}{l}\text { The slope of the rising section of } \\
\text { MFD ( } \mathrm{p}-\mathrm{MFD})\end{array}$} & Increase & $\begin{array}{l}\text { The efficiency of transporting vehicles (passengers) improves } \\
\text { when the road network is in the smooth state }\end{array}$ \\
\hline & & Decrease & The efficiency of transporting vehicles (passengers) \\
\hline & & & $\begin{array}{l}\text { deteriorates when the road network is in the smooth state } \\
\text { The efficiency of transporting vehicles (passengers) and the }\end{array}$ \\
\hline & \multirow{3}{*}{$\begin{array}{l}\text { The value of the stable section of } \\
\text { MFD ( } \mathrm{p} \text {-MFD) }\end{array}$} & Increase & $\begin{array}{l}\text { ability to endure the access of vehicles improve when the } \\
\text { road network is in the saturated state }\end{array}$ \\
\hline & & & The efficiency of transporting vehicles (passengers) and the \\
\hline & & Decrease & $\begin{array}{l}\text { ability to endure the access of vehicles deteriorate when the } \\
\text { road network is in the saturated state }\end{array}$ \\
\hline & \multirow{3}{*}{$\begin{array}{l}\text { The absolute value of the slope of the } \\
\text { descending section of MFD (p- } \\
\text { MFD) }\end{array}$} & & The efficiency of transporting vehicles (passengers) improves \\
\hline & & Increase & when the road network is in the congested state \\
\hline & & Decrease & $\begin{array}{l}\text { The efficiency of transporting vehicles (passengers) } \\
\text { deteriorates when the road network is in the congested state }\end{array}$ \\
\hline & \multirow{3}{*}{$\begin{array}{l}\text { The absolute value of the post-stable } \\
\text { section of MFD (p-MFD) }\end{array}$} & Increase & $\begin{array}{l}\text { The efficiency of transporting vehicles (passengers) improves } \\
\text { when the road network is in the post-congested state }\end{array}$ \\
\hline & & & The efficiency of transporting vehicles (passengers) \\
\hline & & Decrease & $\begin{array}{l}\text { deteriorates when the road network is in the post-congested } \\
\text { state }\end{array}$ \\
\hline \multirow{7}{*}{$\begin{array}{l}\text { Road network } \\
\text { capacity and } \\
\text { reliability }\end{array}$} & \multirow{2}{*}{$\begin{array}{l}\text { The inflection point of } \\
\text { MFD (p-MFD) }\end{array}$} & $\begin{array}{l}\text { Move toward } \\
\quad(0,0)\end{array}$ & $\begin{array}{c}\text { The utilization of road network deteriorates resources from } \\
\text { vehicles (passengers) perspective }\end{array}$ \\
\hline & & $\begin{array}{l}\text { Move away } \\
\text { from }(0,0)\end{array}$ & $\begin{array}{c}\text { The utilization of road network resources improves from } \\
\text { vehicles (passengers) perspective }\end{array}$ \\
\hline & \multirow{3}{*}{ The density range of MFD (p-MFD) } & & The continuity of the road network at this state improves \\
\hline & & Increas & from vehicles (passengers) perspective \\
\hline & & Decrease & $\begin{array}{l}\text { The continuity of the road network at this state deteriorates } \\
\text { from vehicles (passengers) perspective }\end{array}$ \\
\hline & \multirow{2}{*}{ The goodness of fit of MFD (p-MFD) } & Incr & $\begin{array}{c}\text { The stability of the road network improves from vehicles } \\
\text { (passengers) perspective }\end{array}$ \\
\hline & & & $\begin{array}{c}\text { The stability of the road network deteriorates from vehicles } \\
\text { (passengers) perspective }\end{array}$ \\
\hline
\end{tabular}

meters wide. The secondary roads are 2,100 meters long with four lanes, and each lane is 3.5 meters wide. The main roads and the secondary roads are both two-way roads. The branch roads are 100 meters long. Two pairs of harbour-style bus stops are set up on each main road. There are two types of vehicles in the road network, cars and buses. In this experiment, the dedicated bus lanes are set on the roadside. The schematic diagram of the dedicated bus lanes and road network are shown in Figures 5 and 6, respectively. In order to facilitate simulation, bus lanes are only set up on the main roads, and the average bus flow on the dedicated bus lanes is set as $75 \mathrm{veh} / \mathrm{ln} / \mathrm{h}$ based on the field investigation of the average interval on dedicated bus lanes.

The network traffic data under the different scenarios are obtained by a microscopic traffic simulation software of PTV Vissim. This study has simulated five scenarios with different proportions of dedicated bus lanes, which are $0,0.05,0.11$, 0.16 , and 0.21 .

In the simulation network, data detectors are installed at the road network boundary, internal entrances, and exits. The data collection time interval is $60 \mathrm{~s}$, and the total duration of one simulation is $10,500 \mathrm{~s}$. To study the efficiency and capacity of the network comprehensively, cars are continuously input with the time increment of $\Delta_{t}$ in each scenario. By continuously increasing the input of vehicles in the road network, the number of vehicles running on the road network increases continuously. As the density of vehicles in the road network increases, the network traffic state ranges from a smooth state to a saturated state, then congested state, and finally severe congestion state. Therefore, one can collect the traffic parameters from the smooth state to the severely congested state for MFD and p-MFD modelling. In this paper, $\Delta_{t}$ is set to $200 \mathrm{~s}$, and the car arrival patterns on each grade of the road are shown in Table 3 . The flow of cars entering the road network increases according to a certain pattern, which makes the road network transit to a severely congested state from a smooth state. The number of vehicles entering and leaving the road network at each interval during the simulation period is obtained. Five simulations are run for each scenario by changing the random seed such as to reduce the random errors.

In this experiment, $n_{c}=1.3$, and $n_{b}=72$. Notably, for a realistic purpose, the value of $n_{c}$ in this paper is set as the observed average occupancy of cars, similar to reference [28]. The values of $n_{c}$ and $n_{b}$ can be determined through field investigation in practice. Every set of data is processed by equations (1)-(3) and (4)-(7). The four-segment linear function model of MFD, car p-MFD, and bus p-MFD under different scenarios are obtained by the modelling methods proposed, and then road network p-MFD is obtained by equation (12). Finally, the characteristic parameters of the road network MFD and p-MFD are analysed. 


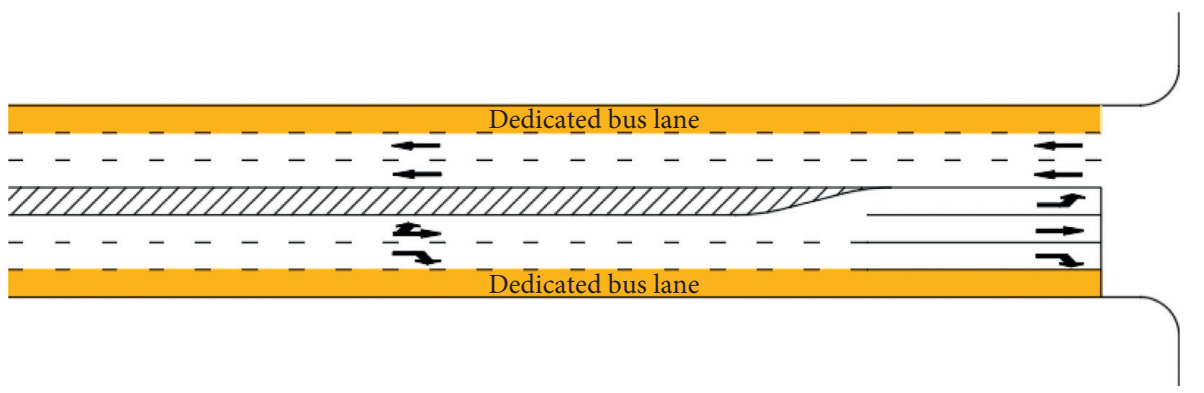

Figure 5: Schematic diagram of dedicated bus lanes.

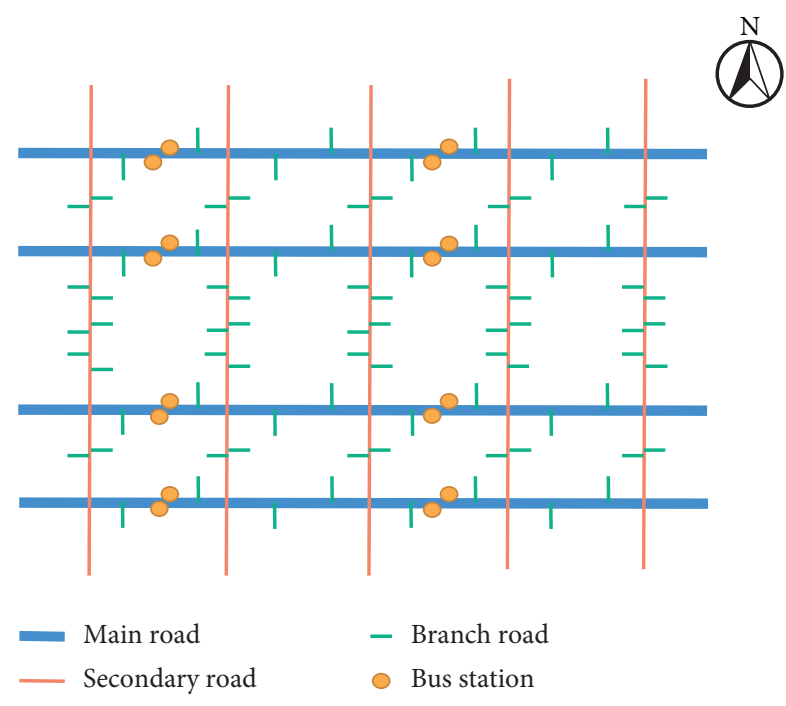

FiguRE 6: Experimental grid road network.

TABle 3: Traffic arrival pattern in the experimental simulation network.

\begin{tabular}{lcc}
\hline Road grade & $\begin{array}{c}\text { Increase of car flow } \\
\text { (veh) }\end{array}$ & $\begin{array}{c}\text { Maximum value of car flow } \\
\text { (veh/ln/h) }\end{array}$ \\
\hline $\begin{array}{l}\text { Main road } \\
\text { Secondary }\end{array}$ & 100 & 1,500 \\
road & 50 & 800 \\
Branch road & 10 & $150 \sim 300$ \\
\hline
\end{tabular}

In addition, the values of $n_{b}$ is related to the average number of passengers carried by buses. It mainly affects bus p-MFD, which reflects the network performance of carrying bus passengers. Compared with car passenger occupancy, the value and fluctuation of bus passenger occupancy are greater, which has a more significant impact on p-MFD. Therefore, taking the proportion of dedicated bus lanes as 0.11 as an example, the bus p-MFD and network p-MFD with the values of $n_{b}$ changing from 10 to 90 are analysed.

\subsection{Experimental Results}

4.2.1. MFD. When the dedicated bus lane proportion is 0.11 , the MFD is taken as an example to show the modelling result of MFD, as shown in Figure 7(a). Figures 7(b) and 7(c) show the comparisons of the MFD linear functions under the different proportions of dedicated bus lanes. Table 4 shows the segmented expressions of MFD with different proportions of dedicated bus lanes obtained by GMM clustering and linear fitting.

In Figure 7, it can be seen that the road network has not reached the final paralysis point at the end of the simulation, which means the flow of vehicles leaving the road network is not 0 . Although the entire road network has serious congestion, and most vehicles cannot leave the road network, there are still a small number of vehicles near the exits that can still leave the road network. Figures 7 (b) and 7(c) show the trend of MFD as the proportion of dedicated bus lanes increases. With the increase of the proportion of dedicated bus lanes, the slope of the rising section gradually increases, and the front- and the back-inflection points gradually move toward zero. The difference in the value of the post-stable section is not obvious. The indicators proposed in Section 3 on MFD are analysed in detail in Section 5.

4.2.2. $p$-MFD. The car $\mathrm{p}-\mathrm{MFD}$, bus $\mathrm{p}-\mathrm{MFD}$, and road network $\mathrm{p}$-MFD under the proportion of 0.11 of dedicated bus lanes are taken as the example to show the modelling result of p-MFD, as shown in Figures $8(a)-8(c)$. It can be seen that both car p-MFD and bus p-MFD have consistent curve shape of rising, stable, descending, and finally stable trends in Figures 8(a) and 8(b). However, there are many differences in the inflection points, peak value, and slopes. Tables 5 and 6 show the segmented expressions of car p-MFD and bus p-MFD with different proportions of dedicated bus lanes obtained by GMM clustering and linear fitting.

According to Figure 8(c), there are three different inflection points for the car p-MFD and the bus p-MFD. Therefore, the road network p-MFD by equation (12) has six inflection points with a seven-segment linear function expression. The rising section $\left(g_{p}>0\right)$ has two segments; the descending section $\left(g_{p}<0\right)$ has three; and the stable and the post-stable sections $\left(g_{p}=0\right)$ both have one segment. This shows that the number of network p-MFD segments is determined by the inflection points of the car p-MFD and the bus $\mathrm{p}-\mathrm{MFD}$, which has a certain degree of uncertainty. However, the general trend of the road network p-MFD is still rising and stable, descending until it reaches a plateau near the minimum value. The rising and descending sections may have multiple segments, which is determined by the 


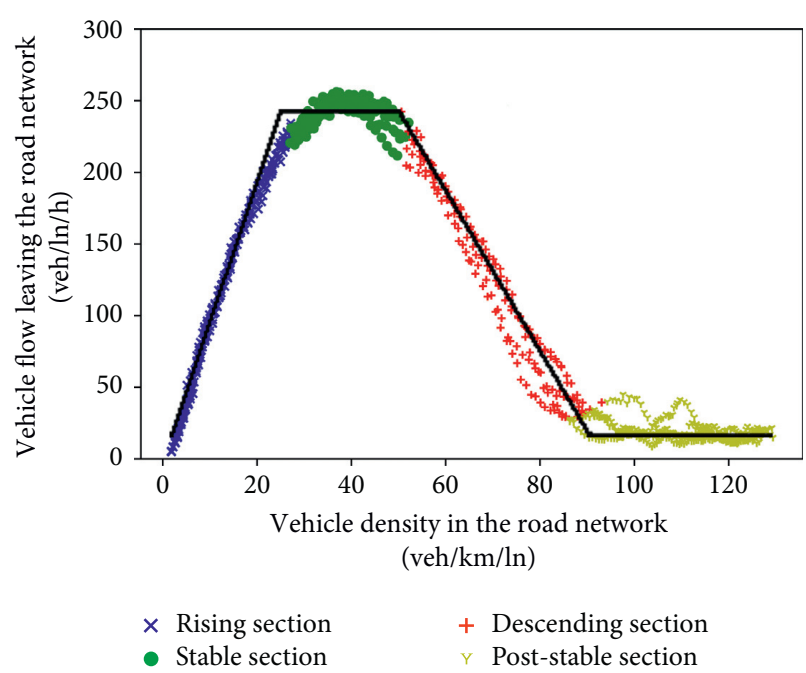

(a)
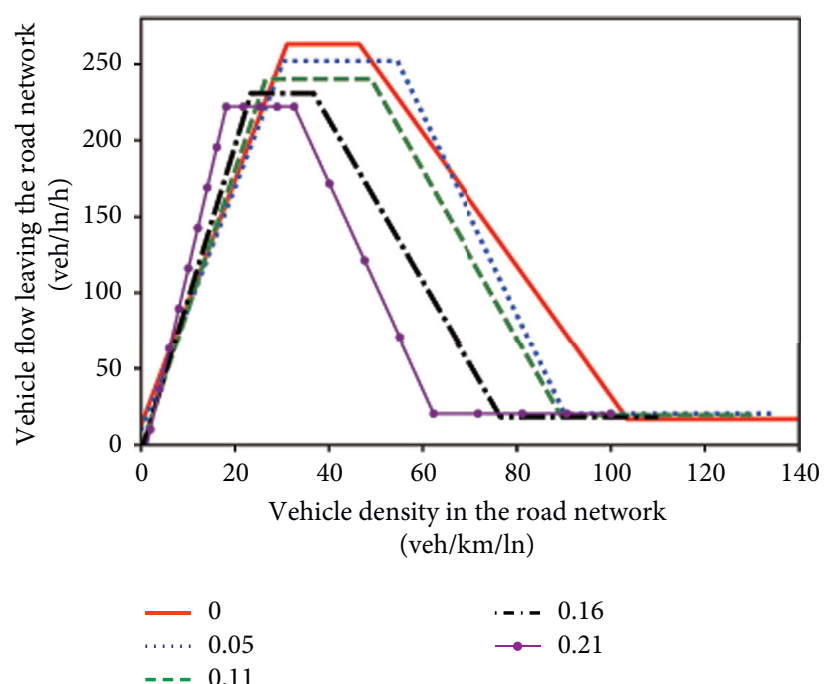

(b)

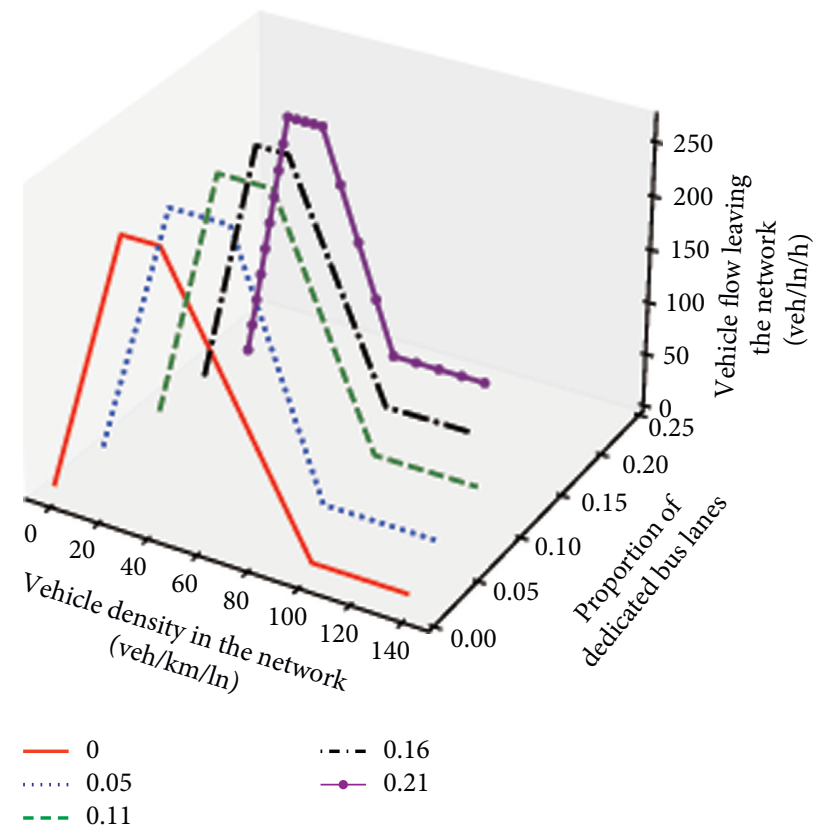

(c)

FIGURE 7: (a) MFD cluster fitting results with dedicated bus lane proportion of 0.11 , (b) MFD with different proportions of dedicated bus lanes, and (c) MFD with different proportions of dedicated bus lanes.

TABLE 4: MFD expressions with different proportions of dedicated bus lanes.

\begin{tabular}{lccccc}
\hline Proportion of dedicated bus lanes & 0 & 0.05 & 0.11 & 0.16 & 0.21 \\
\hline Rising section & $8.094 \mathrm{k}+12.650$ & $8.032 \mathrm{k}+9.511$ & $9.169 \mathrm{k}-2.224$ & $10.133 \mathrm{k}-5.309$ & $13.201 \mathrm{k}-16.332$ \\
Stable section & 262.915 & 252.181 & 240.344 & 230.968 & 222.027 \\
Descending section & $-4.301 \mathrm{k}+461.961$ & $-6.470 \mathrm{k}+603.528$ & $-5.451 \mathrm{k}+505.999$ & $-5.326 \mathrm{k}+425.497$ & $-6.755 \mathrm{k}+441.243$ \\
Post-stable section & 16.955 & 20.477 & 19.398 & 18.283 & 20.423 \\
\hline
\end{tabular}

speed of the car p-MFD and the bus p-MFD entering and leaving the rising and descending sections, respectively. Notably, when the proportion of dedicated bus lanes is 0.21 , all buses in the road network can freely enter and exit the road network through dedicated bus lanes. Therefore, the bus $\mathrm{p}$-MFD has only the rising and the stable sections.
The road network $\mathrm{p}$-MFD with different proportions of dedicated bus lanes is obtained by using equation (12) and the segmented expression of p-MFD for cars and buses. Figures $8(\mathrm{~d})$ and $8(\mathrm{e})$ show the comparison of the linear function models of $\mathrm{p}$-MFD with different proportions of dedicated bus lanes. From the p-MFD obtained, when the 

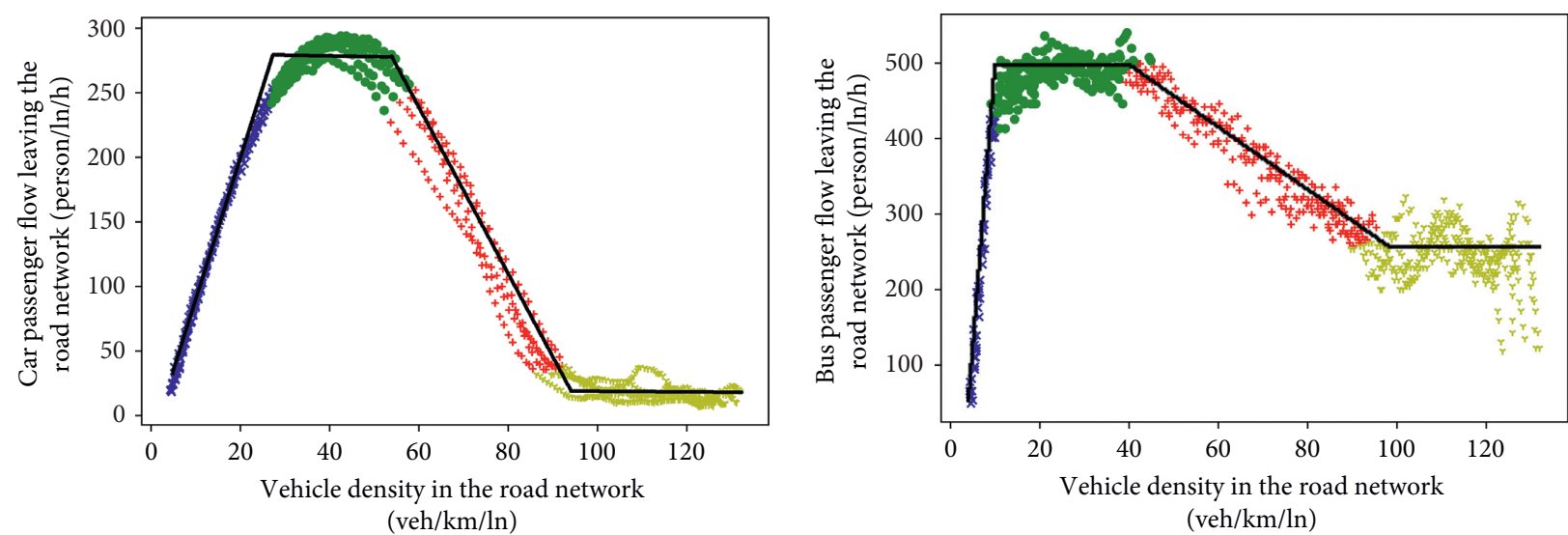

$(\mathrm{veh} / \mathrm{km} / \mathrm{ln})$

$\begin{array}{ll}\times \text { Rising section } & + \text { Descending section } \\ \text { Stable section } & \text { y Post-stable section }\end{array}$

(a)

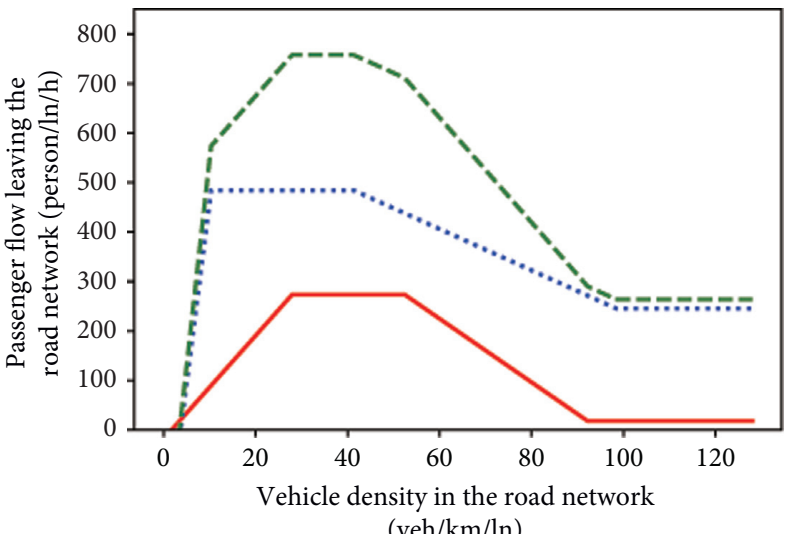

$(\mathrm{veh} / \mathrm{km} / \mathrm{ln})$
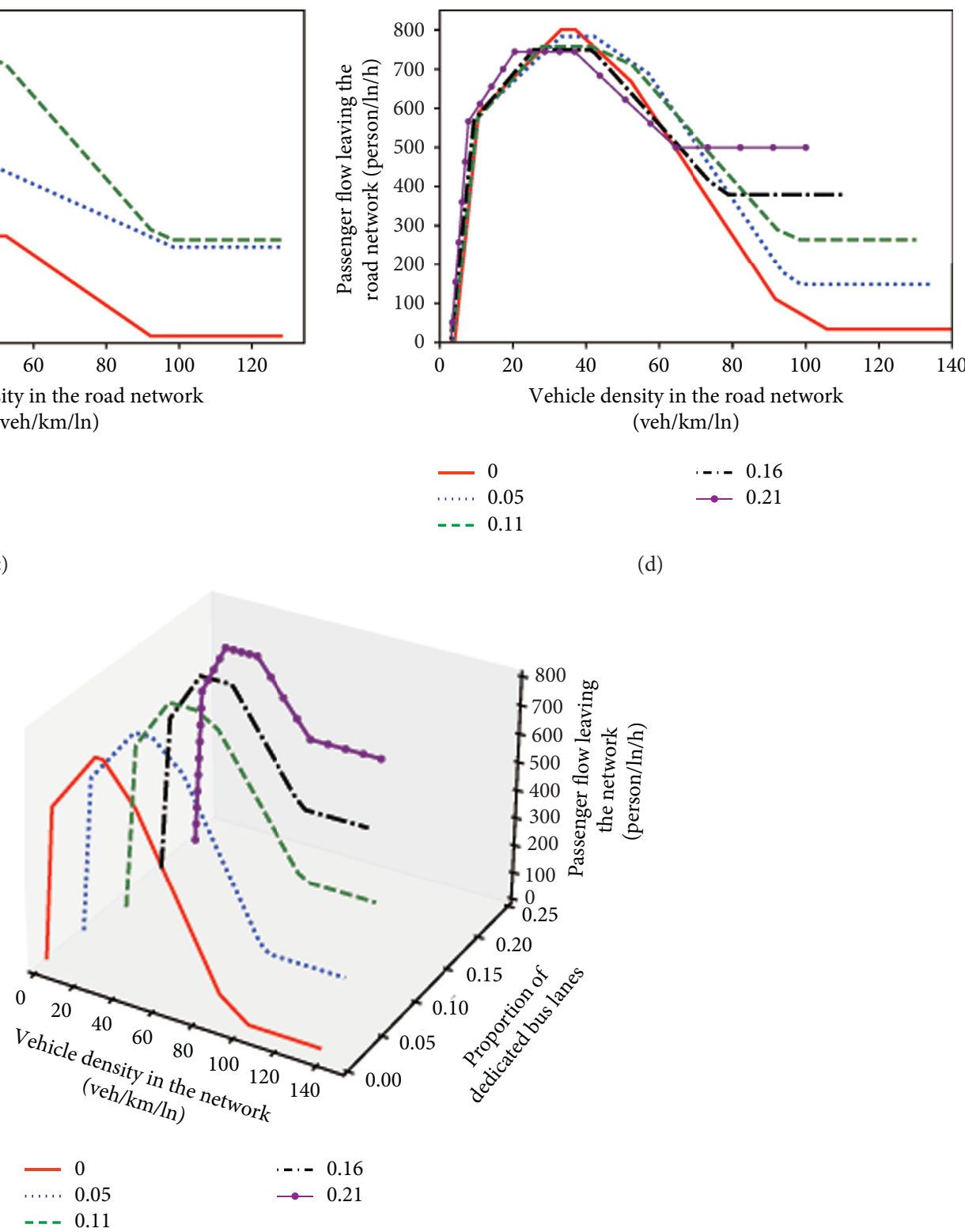

(d)

(e)

Figure 8: (a) p-MFD cluster fitting results of cars with dedicated bus lane proportion of 0.11 , (b) p-MFD cluster fitting results of buses with bus lane proportion of 0.11 , (c) p-MFD of road network with dedicated bus lane proportion of 0.11 , (d) p-MFD with different proportions of dedicated bus lanes, and (e) p-MFD with different proportions of dedicated bus lanes. 
TABle 5: Car p-MFD expression with different proportions of dedicated bus lanes.

\begin{tabular}{lccccc}
\hline Proportion of dedicated bus lanes & 0 & 0.05 & 0.11 & 0.16 & 0.21 \\
\hline Rising section & $9.436 \mathrm{k}-0.132$ & $8.819 \mathrm{k}+3.010$ & $10.428 \mathrm{k}-17.927$ & $11.140 \mathrm{k}-17.496$ & $14.101 \mathrm{k}-27.883$ \\
Stable section & 311.952 & 295.718 & 273.427 & 267.126 & 260.573 \\
Descending section & $-5.421 \mathrm{k}+594.830$ & $-7.473 \mathrm{k}+721.232$ & $-6.419 \mathrm{k}+609.990$ & $-6.673 \mathrm{k}+541.792$ & $-8.861 \mathrm{k}+586.643$ \\
Post-stable section & 20.890 & 21.754 & 18.479 & 14.609 & 15.780 \\
\hline
\end{tabular}

TABLE 6: Bus p-MFD expression with different proportions of dedicated bus lanes.

\begin{tabular}{lccccc}
\hline Proportion of dedicated bus lanes & 0 & 0.05 & 0.11 & 0.16 & 0.21 \\
\hline Rising section & $80.497 \mathrm{k}-365.479$ & $76.460 \mathrm{k}-319.657$ & $73.042 \mathrm{k}-270.203$ & $78.423 \mathrm{k}-252.100$ & $104.309 \mathrm{k}-333.356$ \\
Stable section & 488.711 & 487.780 & 484.173 & 481.680 & 483.791 \\
Descending section & $-8.715 \mathrm{k}+811.437$ & $-6.389 \mathrm{k}+756.586$ & $-4.182 \mathrm{k}+657.039$ & $-3.765 \mathrm{k}+641.574$ & - \\
Post-stable section & 12.843 & 126.845 & 245.322 & 364.262 & - \\
\hline
\end{tabular}

proportion of dedicated bus lanes is no greater than 0.16 , the road network $\mathrm{p}$-MFD has a seven-segment linear function; the rising section $\left(g_{p}>0\right)$ has two segments; and the stable and the post-stable sections have one segment, and the descending section $\left(g_{p}<0\right)$ has three segments. When the proportion of dedicated bus lanes is 0.21 , the road network $\mathrm{p}$-MFD is a five-segment linear function; the rising section $\left(g_{p}>0\right)$ has two segments; the stable and the post-stable sections have one segment, and the descending section $\left(g_{p}<0\right)$ has also one section. The indicators proposed in Section 3 on p-MFD are analysed in detail in Section 6.

4.2.3. Impact Analysis of $n_{b}$ on $p-M F D$. The bus $\mathrm{p}-\mathrm{MFD}$ and network p-MFD with the values of $n_{b}$ changing from 10 to 90 when the proportion of dedicated bus lanes is 0.11 are taken as the example to demonstrate the impact of $n_{b}$ on p-MFD, as shown in Figures 9(a) and 9(b). When the average passenger occupancy of buses in the road network increases, the basic shapes of the bus p-MFD and the network p-MFD remain unchanged. It shows that with the increase of the vehicle density, the passenger flow first increases, then stabilizes, and then drops to a certain small value.

For bus p-MFD and network p-MFD, when the values of $n_{b}$ increases, changing from 10 to 90 , the slopes of the rising section, the values of the stable section, and the post-stable section become larger. According to the indicators based on the $\mathrm{p}-\mathrm{MFD}$, it means that when the number of passengers carried by buses in the network becomes larger, the efficiency of transporting passengers improves.

This shows that when the average passenger occupancy of cars in the network is certain, appropriately increasing the average passenger occupancy of buses can improve the transport efficiency of the road network. The optimization of the network transport efficiency can be realized from two aspects. On the one hand, the passenger capacity of buses should be improved, and the carriages should be rationally optimized to increase the number of passengers that can be accommodated. On the other hand, the level of public transportation services should be improved to increase the attractiveness of public transportation.

\section{Impact Analysis of Dedicated Bus Lanes on MFD}

5.1. Geometric Features. The proportion of dedicated bus lanes affects the geometric characteristics of MFD, mainly including the rising section, the descending section, the stable section, the points of inflection, and the density range of each section.

5.1.1. The Rising and Descending Sections. The absolute values of the slopes of the rising and the descending sections of the MFD under different proportions of dedicated bus lanes are compared as shown in Figure 10. In general, as the proportion of dedicated bus lanes in the road network increases from 0 to 0.21 , the slope of the rising section increases. However, the absolute value of the slope of the descending section is less affected. It indicates that the implementation of dedicated bus lanes is conducive to the transport of vehicles in the smooth state of the road network, and as the proportion of dedicated bus lanes increases, this advantage becomes more obvious. When traffic flow in the road network is small, the vehicles in the road network are in a noncongested state. Cars and buses driving in separate lanes can ensure that their speeds are maintained within the ideal range, thereby improving the overall transport efficiency of the road network. Meanwhile, the implementation of dedicated bus lanes has little effect on network efficiency when the road network is congested.

5.1.2. The Stable Section. The values of the stable section $q_{\max }$ with different proportions of dedicated bus lanes are compared as shown in Figure 11. As the proportion of dedicated bus lanes increases from 0 to 0.21 , the value of the stable section decreases. It indicates that the implementation of dedicated bus lanes reduces the capacity of transporting vehicles when the road network is in a saturated state. The impact is more significant with the increase in the proportion of dedicated bus lanes. Due to the limited road resources, the increase in the number of dedicated bus lanes will cause the reduction of the number of passenger-car 

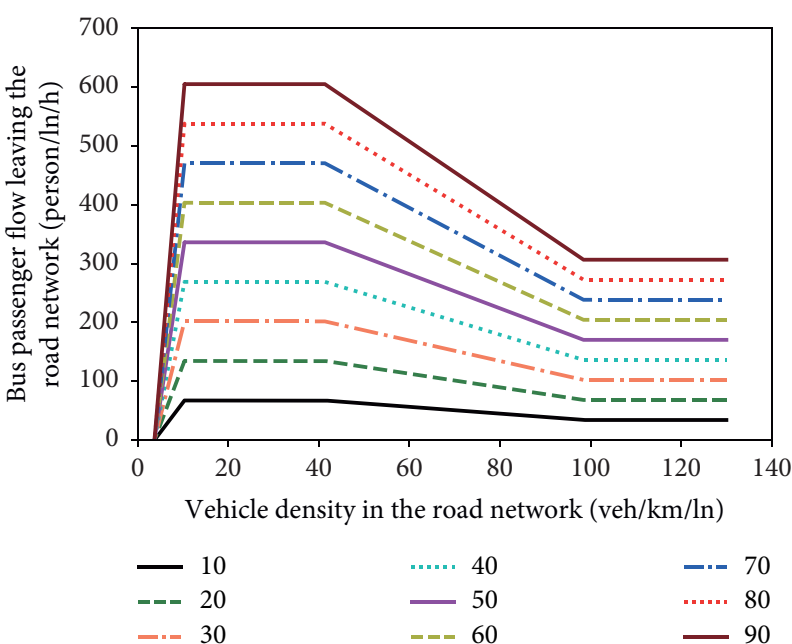

(a)

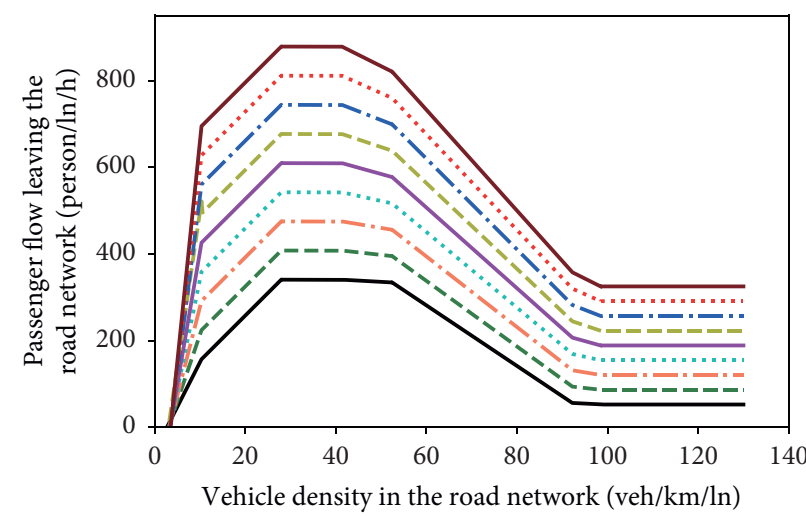

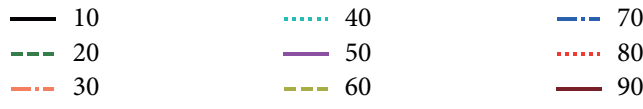

(b)

FIgURE 9: (a) Bus p-MFD with different $n_{b}$ (person/veh) and (b) network p-MFD with different $n_{b}$ (person/veh).

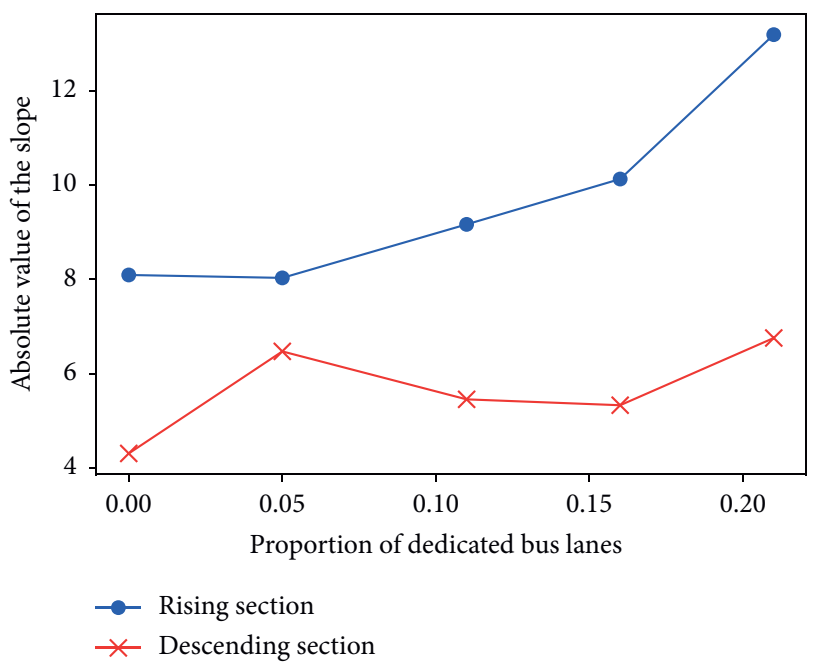

Figure 10: The slope of MFD under the different proportions of dedicated bus lanes.

lanes. The number of buses running in the dedicated bus lane is related to the setting of the bus lanes, the bus departure interval, and so on. Due to its transport characteristics and public service attributes, the output flow of the dedicated bus lanes is less than the output flow of the passenger-car lanes. Therefore, the implementation of dedicated bus lanes reduces the number of vehicles that can be served by the road network.

5.1.3. The Points of Inflection. The horizontal and vertical coordinates of the inflection points of the MFD with different proportions of dedicated bus lanes are compared as shown in Figure 12. As the proportion of dedicated bus lanes increases from 0 to 0.21 , the values of $k_{b}^{s}$ and $q_{\max }$ both show a decreasing trend, and the value of $k_{b}^{d}$ first increases and then decreases. The position of the front-inflection point moves roughly toward the zero point. It shows that the

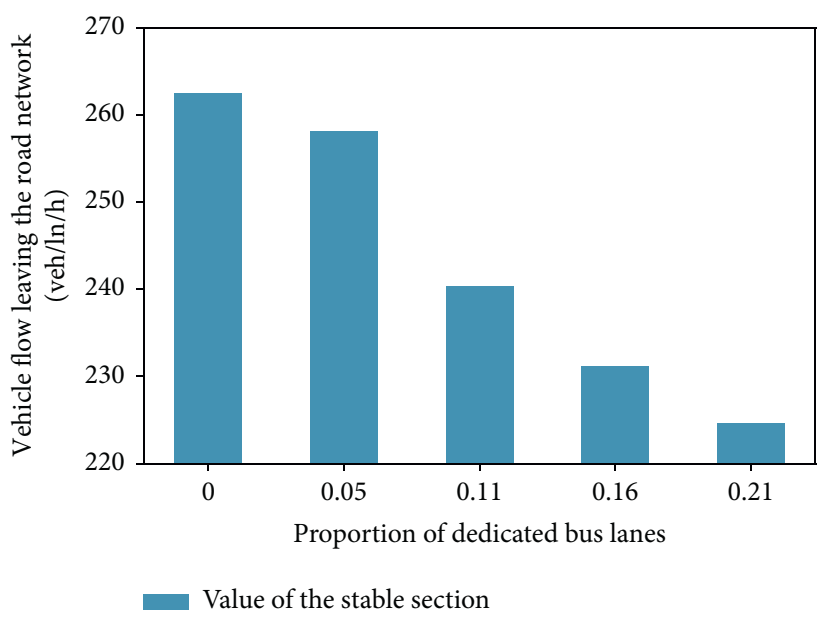

FIGURE 11: Value of the stable section of MFD with different proportions of bus lanes.

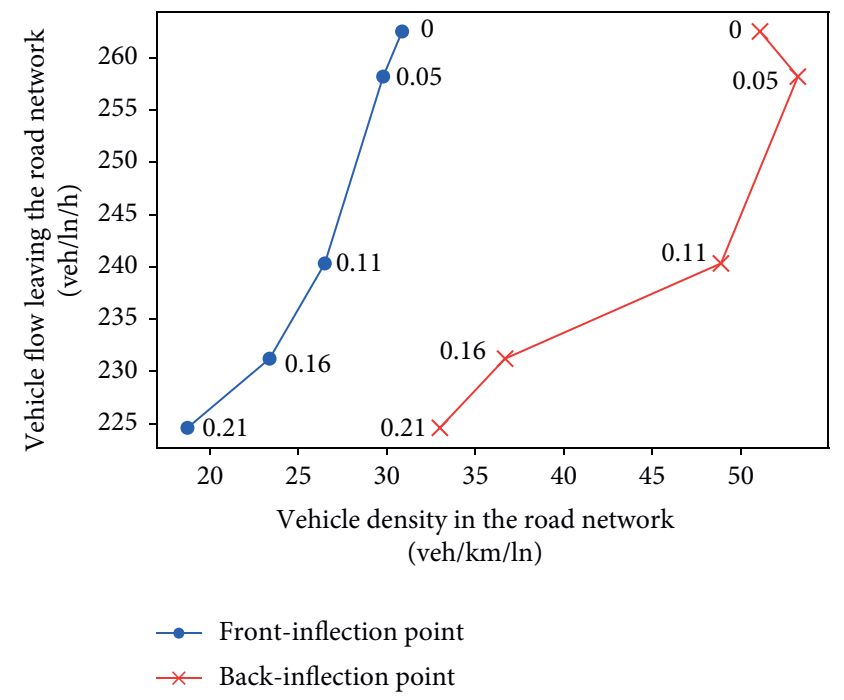

FIGURE 12: Inflection point of MFD with different proportions of dedicated bus lanes. 
implementation of dedicated bus lanes makes the road network transit from the smooth state to the saturated state faster, and as the proportion of dedicated bus lanes increases, the changing speed of the transport state increases. The abscissa of the back-inflection point first increases and then decreases, indicating that when the proportion of dedicated bus lanes is set in the range of $0 \sim 0.11$, the speed at which the road network enters congestion will slow down. As the proportion of dedicated bus lanes continues to increase, the road network will quickly change from a saturated state to a congested state.

The implementation of dedicated bus lanes in the road network causes the reduction of the number of passengercar lanes and also the access of vehicles that the road network can withstand. When the proportion of dedicated bus lanes is in the range of $0 \sim 0.11$, buses and cars running in different lanes effectively reduce the influence of each other. It is beneficial to increase the number of vehicles effectively borne by the road network. However, as the proportion of dedicated bus lanes continues to increase, passenger-car lanes are reduced more, and the number of vehicles effectively borne by the road network decreases sharply.

5.1.4. The Density Range of Each Section. The density range of each section $\Delta k_{\text {range }}^{x}$ with different proportions of dedicated bus lanes is compared as shown in Figure 13. When the proportion of dedicated bus lanes in the road network is within the range of $0 \sim 0.11, \Delta k_{\text {range }}^{s}$ and $k_{b}^{d}$ are relatively large, respectively. It means the road network can effectively bear more vehicles and maintain high-efficiency operation in a long density range. In addition, it can be seen that the implementation of dedicated bus lanes has led to a decrease in traffic density on the road network, which indicates that the traffic density on dedicated bus lanes is less than that on the normal lanes. The reason is that dedicated bus lanes can maintain a certain driving speed. However, the provision of dedicated bus lanes reduces the utilization of road resources by vehicles to a certain extent.

5.2. Goodness of Fit. The proportion of dedicated bus lanes affects the clustering of MFD scattered points, and the goodness of fit is studied.

The goodness of fit of the rising section and that of the descending section, $R^{2}$, with different proportions of dedicated bus lanes are compared as shown in Figure 14. As the proportion of dedicated bus lanes increases from 0 to 0.21 , the $R^{2}$ of the rising and the descending sections shows an increasing trend. The scatter points of the MFD with a certain proportion of dedicated bus lanes are more concentrated than the ones with no dedicated bus lanes. The implementation of dedicated bus lanes is conducive to maintain the stability of road network operation. The main reason is that the dedicated bus lanes allow buses and cars to drive in different lanes, reducing the mutual influence caused by the difference in operating characteristics between them, thereby improving the stability of road network operation.

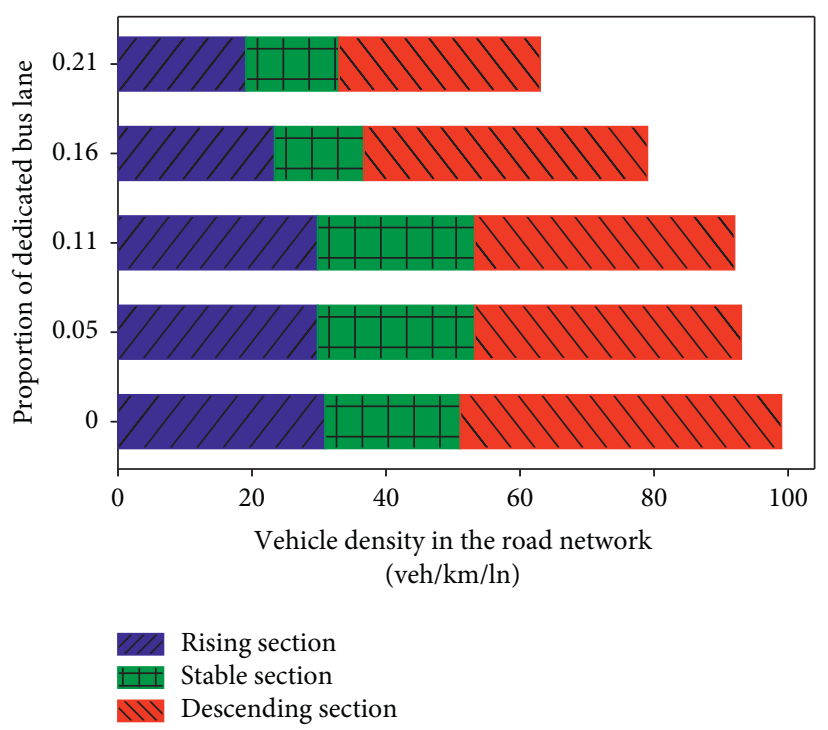

FIGURE 13: Density range of MFD with different proportions of bus lanes.

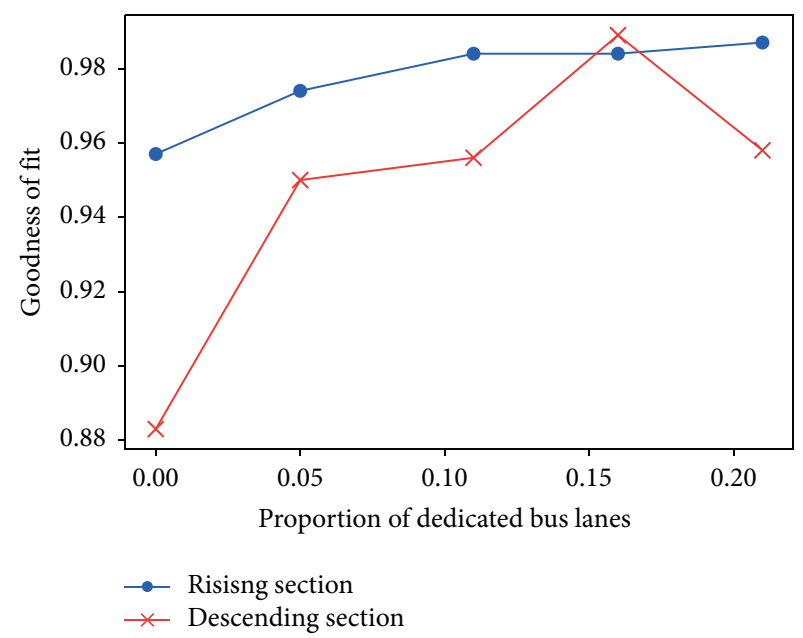

FIGURE 14: $R^{2}$ of MFD with different proportions of dedicated bus lanes.

In summary, the proportion of dedicated bus lanes has certain influences on MFD, especially from the following perspective: the slopes of the rising section, the value of the stable section, the inflection points, density range, and goodness of fit. The impacts of the proportion of dedicated bus lanes on the slopes of the descending section and the value of the post-stable section are not obvious.

\section{Impact Analysis of Dedicated Bus Lanes on p-MFD}

The proportion of dedicated bus lanes affects the geometric characteristics of $\mathrm{p}-\mathrm{MFD}$, mainly including the rising, the descending, the stable and the post-stable sections.

6.1. The Rising and the Descending Sections. The slopes of the rising section of the p-MFD with different dedicated bus 
lanes proportions are compared as shown in Figure 15. The slope of the first segment belonging to the rising section is greater than the second, indicating that there are two stages in the process of transporting passengers when the road network is in a smooth state. According to equation (13), the slope of the p-MFD rising section is the sum of the slopes of the rising section of the corresponding car p-MFD and the bus p-MFD. When $0<k<\min \left\{k_{b}^{1}, k_{c}^{1}\right\}$, the bus p-MFD enters the stable section first since $k_{c}^{1}>k_{b}^{1}$. At this time, $g_{p b}(k)=0$, so the slope of the second segment that belongs to the rising section of $\mathrm{p}$-MFD is equal to the slope of car p-MFD. This shows that in the first stage, with the continuous input of vehicles, passenger transport of cars and buses is in a smooth state, and the passenger transport efficiency reaches the maximum. In the second stage, as the density of vehicles in the road network continues to increase, bus passenger transport reaches saturation first. At this time, the flow of the road network to transport passengers continues to increase. It is worth noting that the speed at which the passenger transport of buses reaches saturation is related to the interval between bus departures and the number of bus routes on dedicated bus lanes.

The absolute slopes of the p-MFD descending section with different dedicated bus lanes proportions are compared as shown in Figure 16. The slope value of the descending section changes twice, indicating that there are three stages in the process of transporting passengers when the road network is in a congested state.

When the proportion of bus lanes is 0 , the slope of the first stage corresponds to that of the bus p-MFD. The slope of the second stage is corresponding to the sum of the slope of the descending section of the car $\mathrm{p}-\mathrm{MFD}$ and the bus $\mathrm{p}-\mathrm{MFD}$. The slope of the third stage is corresponding to that of the car p-MFD. This shows that when the road network is not implemented with dedicated bus lanes, in the first stage, with the continuous input of vehicles, some intersections and road sections on the road network will be blocked, and the passenger transport of the bus first reaches the congested state. At this time, the flow of the road network to transport passengers continues to decline, and the efficiency of bus passenger transport has slowed down. In the second stage, as the traffic density continues to increase, both bus and car passenger transports have reached the congested state. At this time, the passenger flow of the road network continues to drop, and the efficiency of the passenger transport is significantly reduced. In the third stage, the passenger transport capacity of buses is reduced to the minimum, and most bus passengers cannot leave the road network. At this time, the flow of the road network to transport passengers continues to decline.

If the proportion of dedicated bus lanes is greater than 0 , the slope of the first and third stages of the descending section corresponds to that of the bus, and the slope of the second stage of the p-MFD descending section is the sum of the slopes of the descending sections of the corresponding car p-MFD and the bus p-MFD. It indicates that the passenger transport in the first and second stages of the road network is the same as the transport without dedicated bus lanes. The difference is that in the third stage, the passenger transport capacity of cars is reduced to the minimum, and most car passengers cannot leave the road network. At this

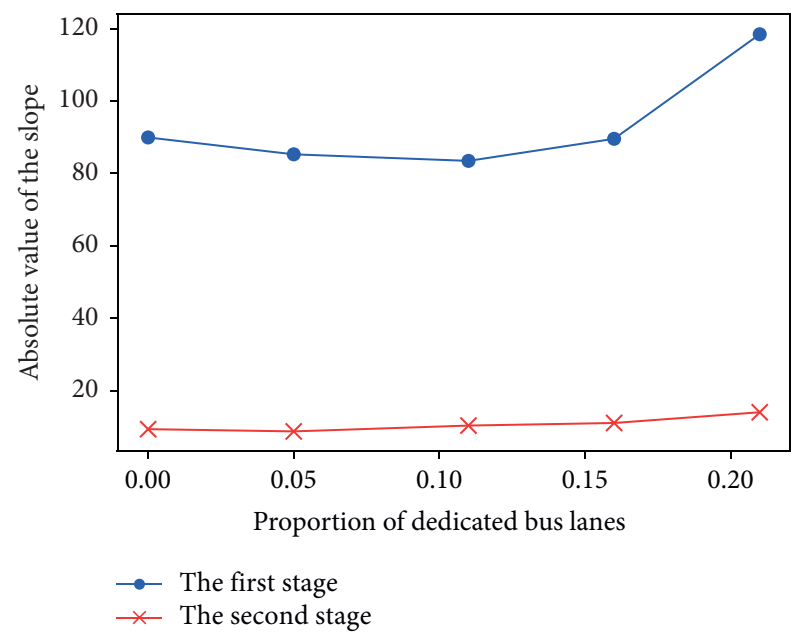

FIgURE 15: Slope of the rising section of p-MFD with different proportions of bus lanes.

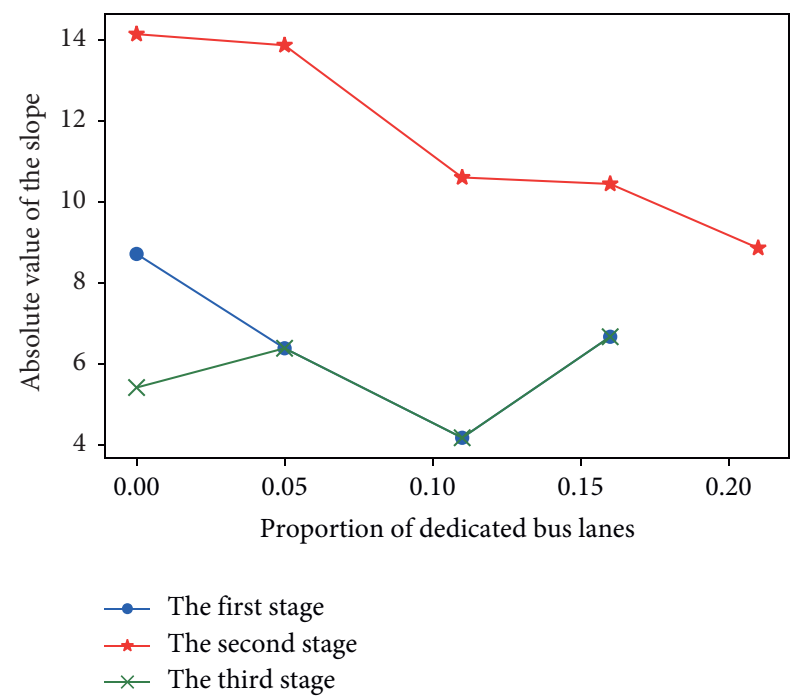

FIGURE 16: Slope of the descending section of p-MFD with different proportions of bus lanes.

time, the flow of the road network to transport passengers continues to decline. This indicates that when the road network is congested, bus passengers can use the dedicated bus lanes to enter quickly and exit the road network, while the serious blockage of passenger-car lanes makes it difficult for car passengers to do so.

It can be seen from Figure 16 that as the proportion of dedicated bus lanes increases from 0 to 0.21 , the absolute value of the slope of the descending section in the second stage has a decreasing trend. It indicates that the dedicated bus lanes improve the transport efficiency of passengers when a road network is in a congested state. As the proportion increases, the impact becomes more significant.

6.2. The Stable Section. The values of the stable section of the p-MFD with different proportions of dedicated bus lanes are compared as shown in Figure 17(a). As the proportion of 


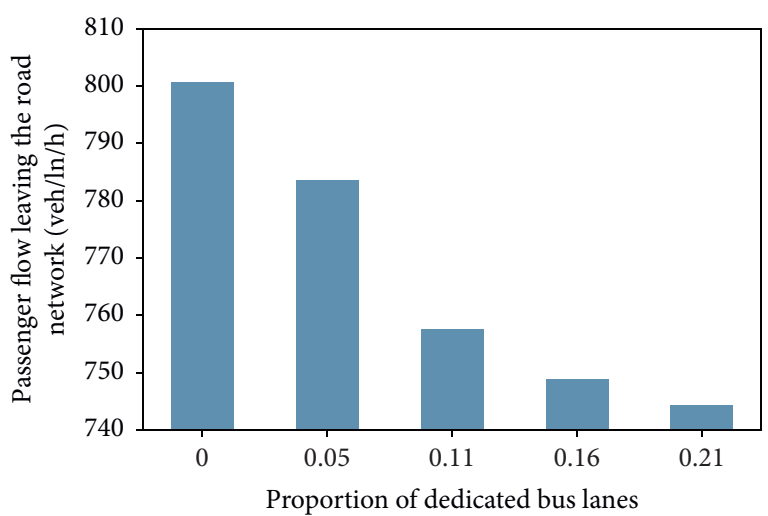

Value of the stable section

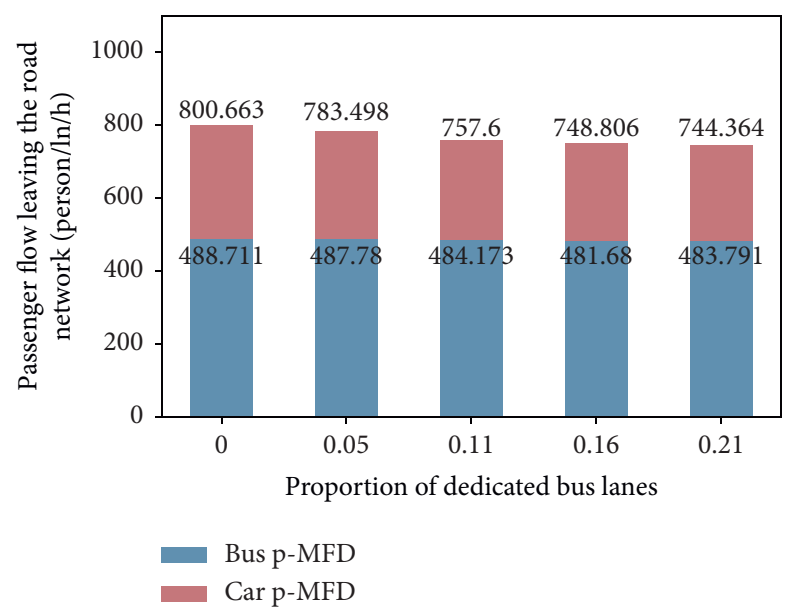

(b)

Figure 17: (a) Value of stable section of p-MFD with different proportions of bus lanes and (b) value of car p-MFD and bus p-MFD stable section with different proportions of bus lanes.

dedicated bus lanes increases from 0 to 0.21 , the value of the stable section continues to decrease. It indicates that dedicated bus lanes reduce the capacity of the road network to transport passengers in a saturated state. The proportion increases, and the impact is more significant.

The values of the stable section of the bus p-MFD and the car p-MFD with different proportions of dedicated bus lanes are compared, as shown in Figure 17(b). It can be seen from Figure 17(b) that as the proportion of dedicated bus lanes increases, the value of the stable section of bus p-MFD fluctuates around 484 persons/lane/h, while the value of the stable section of car $\mathrm{p}$-MFD continues to decrease. This indicates that the dedicated bus lanes reduce the ability of the road network to withstand the entry and exit of car passengers. At this time, dedicated bus lanes do not bring obvious benefits to the road network to transport bus passengers. The main reason is that in this state, the road network transport is able to maintain efficient operation. Most of the roads have not been congested, and at the same time, the passenger transport of buses is saturated according to the bus service level. Therefore, even if there is no dedicated bus lane, buses can still efficiently transport passengers in and out of the road network.

6.3. The Post-Stable Section. The values of the post-stable section of the p-MFD with different proportions of dedicated bus lanes are compared as shown in Figure 18. As the proportion of dedicated bus lanes increases from 0 to 0.21 , the value of the post-stable section continues to increase. It indicates that the dedicated bus lanes improve the ability of the road network to transport passengers in the post-congested state. The impact is more significant when the proportion increases. The main reason is that when the road network is in a post-congested state, most of the intersections and road sections in the road network are severely congested. Without dedicated bus lanes, most car passengers and bus passengers cannot leave the road network. However, the dedicated bus lanes can ensure the passage of buses;

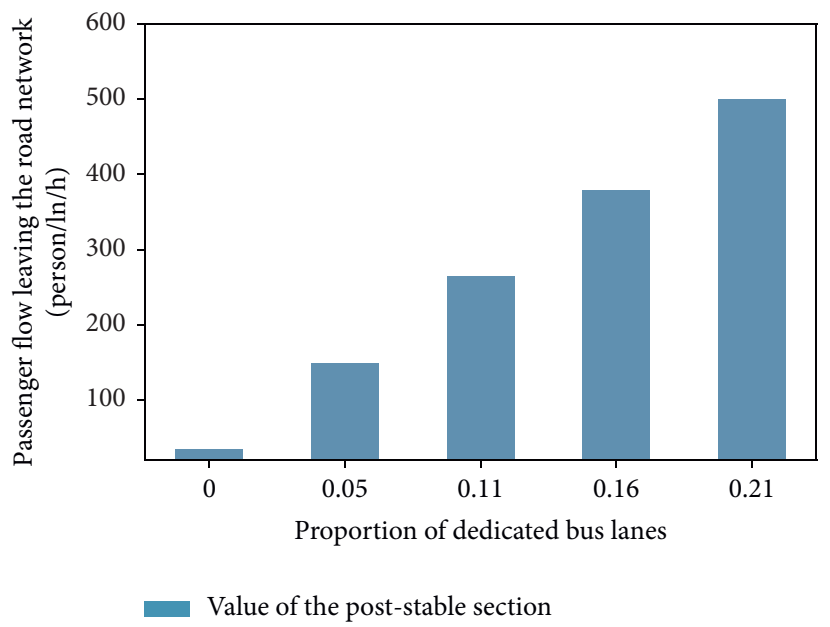

FIgURE 18: Value of p-MFD post-stable section with different proportions of dedicated bus lanes.

therefore, the road network can maintain efficient transport of bus passengers when most roads are blocked.

In summary, the proportion of dedicated bus lanes has certain influences on $\mathrm{p}$-MFD, specifically from the following perspective: the slopes of the rising and the descending sections and the values of the stable and the post-stable sections. Since the number of segments of network p-MFD is flexible, the patterns of the other indicators caused by the impacts of the proportion of dedicated bus lanes are not obvious.

\section{Conclusions}

This paper proposes the description and modelling methods of MFD and p-MFD based on GMM and linear fitting and the corresponding performance indicators of urban road networks. The methodology proposed in this study can be applicable to various scenarios of urban road networks and 
even can be extended to multiple modes (cars, buses, etc.). A grid network is taken as a case study such as to analyse the impact of different dedicated bus lane proportions on network performance via microscopic traffic simulation for MFD and p-MFD modelling. There are some interesting findings as follows:

(i) The larger the proportion of dedicated bus lanes, the more the rising and the stable sections of MFD, and the descending and post-stable sections of $\mathrm{p}-\mathrm{MFD}$ are affected.

(ii) The slope of the MFD rising section tends to increase, and the value of the MFD stable section tends to decrease as the proportion of dedicated bus lanes increases, which indicates that dedicated bus lane improves the efficiency of vehicle transport under the smooth state of the road network. However, it might reduce the vehicle throughput of the road network.

(iii) The slope of the p-MFD descending section and the value of the post-stable section tend to increase when the proportion of dedicated bus lanes is increased. It indicates that dedicated bus lanes improve the efficiency of passenger transport under the saturated state of the road network. The impact is more significant when the proportion grows.

(iv) When the proportion of dedicated bus lanes on the road network is within the range of 0 to 0.11 , the road network can effectively bear more vehicles and maintain a longer density range with efficient operation.

(v) As the proportion of dedicated bus lanes increases, the goodness of fit of the MFD rising and descending sections tend to increase, which indicates that dedicated bus lanes are conducive to improve the transport stability of the road network.

(vi) As the bus passenger occupancy increases, the slopes of the rising section and the values of the stable and the post-stable sections of p-MFD become larger.

To sum up, for the simulated road network, dedicated bus lanes can improve the efficiency of vehicle and passenger transport in the smooth state of the road network, also ensure that the road network continues to carry passengers in the congested state, and improve the stability of the road network transport. However, in the meantime, dedicated bus lanes also affect the ability of the road network to withstand the entry and exit of car passengers. Therefore, when implementing dedicated bus lanes, reasonable consideration should be given to the proportion and the operating period. The operation state of the road network is recommended to be monitored in real-time, and dedicated bus lanes are suggested to be implemented when the road network is in the smooth and the post-congested states. In addition, the proportion of dedicated bus lanes should be reasonably allocated based on bus demand and operation conditions, which provides a reference for better implementation of dedicated bus lanes. Besides, the bus passenger capacity and bus service should be improved to increase the attractiveness of public transportation.

This paper proposes a group of indicators based on MFD and $\mathrm{p}$-MFD and summarizes the influence mechanism of dedicated bus lanes on road network transport in different states, aiming to support and expand existing research. In the future, the influence of different forms of bus lanes, operating periods of bus lanes, as well as the influence of bus signal priority on MFD and p-MFD should be studied to assist traffic planning and management departments to optimize the public transport system further. Meanwhile, it will help increase the attractiveness of public transport and improve the comprehensive transport utility of the road network.

\section{Data Availability}

The data used to support the findings of this study are available from the corresponding author upon request.

\section{Conflicts of Interest}

The authors declare that there is no conflict of interest.

\section{Acknowledgments}

The authors acknowledge Xiaoran Qin from the Hong Kong University of Science and Technology for her valuable comments on this research. This paper was supported by the General Project of the National Natural Science Foundation of China (52072129).

\section{References}

[1] M. Garcia and K. Yamamoto, "Busways and bus lanes in Brazil and Japan," in Proceedings of the VNIS'94-1994 Vehicle Navigation and Information Systems Conference, Yokohama, Japan, August 1994.

[2] Ministry of Transport of the People's Republic of China, Statistical Bulletin on the Development of the Transportation Industry in 2019, 2019.

[3] N. Chiabaut, X. Xie, and L. Leclercq, "Performance analysis for different designs of a multimodal urban arterial," Transportation Business: Transport Dynamics, vol. 2, no. 3, pp. 229-245, 2014.

[4] J. Zhao, J. Yu, X. Xia, J. Ye, and Y. Yuan, "Exclusive bus lane network design: a perspective from intersection operational dynamics," Networks and Spatial Economics, vol. 19, no. 4, pp. 1143-1171, 2019.

[5] C. F. Daganzo, "Urban gridlock: macroscopic modeling and mitigation approaches," Transportation Research Part B: Methodological, vol. 41, no. 1, pp. 49-62, 2007.

[6] N. Geroliminis and C. F. Daganzo, "Existence of urban-scale macroscopic fundamental diagrams: some experimental findings," Transportation Research Part B: Methodological, vol. 42, no. 9, pp. 759-770, 2008.

[7] C. F. Daganzo and N. Geroliminis, "An analytical approximation for the macroscopic fundamental diagram of urban traffic," Transportation Research Part B: Methodological, vol. 42, no. 9, pp. 771-781, 2008. 
[8] M. J. Cassidy, K. Jang, and C. F. Daganzo, "Macroscopic fundamental diagrams for freeway networks: theory and observation," Transportation Research Record: Journal of the Transportation Research Board, vol. 2260, no. 1, pp. 8-15, 2011.

[9] V. L. Knoop and S. P. Hoogendoorn, "Empirics of a generalized macroscopic fundamental diagram for urban freeways," Transportation Research Record: Journal of the Transportation Research Board, vol. 2391, no. 1, pp. 133-141, 2013.

[10] N. Zheng and N. Geroliminis, "On the distribution of urban road space for multimodal congested networks," ProcediaSocial and Behavioral Sciences, vol. 80, pp. 119-138, 2013.

[11] N. Chiabaut, "Evaluation of a multimodal urban arterial: the passenger macroscopic fundamental diagram," Transportation Research Part B: Methodological, vol. 81, pp. 410420, 2015.

[12] A. Loder, L. Ambühl, M. Menendez, and K. W. Axhausen, "Empirics of multi-modal traffic networks-using the 3D macroscopic fundamental diagram," Transportation Research Part C: Emerging Technologies, vol. 82, pp. 88-101, 2017.

[13] C. Buisson and C. Ladier, "Exploring the impact of homogeneity of traffic measurements on the existence of macroscopic fundamental diagrams," Transportation Research Record: Journal of the Transportation Research Board, vol. 2124, no. 1, pp. 127-136, 2009.

[14] J. Haddad and N. Geroliminis, "On the stability of traffic perimeter control in two-region urban cities," Transportation Research Part B: Methodological, vol. 46, no. 9, pp. 1159-1176, 2012.

[15] T. Tsubota, A. Bhaskar, and E. Chung, "Macroscopic fundamental diagram for Brisbane, Australia," Transportation Research Record: Journal of the Transportation Research Board, vol. 2421, no. 1, pp. 12-21, 2014.

[16] C. Huang, N. Zheng, and J. Zhang, "Investigation of bimodal macroscopic fundamental diagrams in large-scale urban networks: empirical study with GPS data for Shenzhen city," Transportation Research Record: Journal of the Transportation Research Board, vol. 2673, no. 6, pp. 114-128, 2019.

[17] E. Saffari, M. Yildirimoglu, and M. Hickman, "A methodology for identifying critical links and estimating macroscopic fundamental diagram in large-scale urban networks," Transportation Research Part C: Emerging Technologies, vol. 119, 2020.

[18] G. Tilg, S. Amini, and F. Busch, "Evaluation of analytical approximation methods for the macroscopic fundamental diagram," Transportation Research Part C: Emerging Technologies, vol. 114, pp. 1-19, 2020.

[19] Z. Y. Gu and M. Saberi, "Simulation-based optimization of toll pricing in large-scale urban networks using the network fundamental diagram: a cross-comparison of methods," Transportation Research Part C: Emerging Technologies, vol. 122, 2021.

[20] Z. Gu, F. Safarighouzhdi, M. Saberi, and T. H. Rashidi, “A macro-micro approach to modeling parking," Transportation Research Part B: Methodological, vol. 147, pp. 220-244, 2021.

[21] Z. Y. Gu, A. Najmi, M. Saberi et al., "Macroscopic parking dynamics modeling and optimal real-time pricing considering cruising-for-parking," Transportation Research Part C: Emerging Technologies, vol. 118, 2020.

[22] I. Dakic, K. Yang, M. Menendez, and J. Y. J. Chow, "On the design of an optimal flexible bus dispatching system with modular bus units: using the three-dimensional macroscopic fundamental diagram," Transportation Research Part B: Methodological, vol. 148, pp. 38-59, 2021.
[23] S. Sirmatel, D. Tsitsokas, A. Kouvelas et al., "Modeling, estimation, and control in large-scale urban road networks with remaining travel distance dynamics," Transportation Research Part C: Emerging Technologies, vol. 128, 2021.

[24] W. Lu, J. Liu, J. Mao, G. Hu, C. Gao, and L. Liu, "Macroscopic fundamental diagram approach to evaluating the performance of regional traffic controls," Transportation Research Record: Journal of the Transportation Research Board, vol. 2674, no. 7, pp. 420-430, 2020.

[25] L. Ambuhl, A. Loder, M. C. J. Bliemer et al., "A functional form with a physical meaning for the macroscopic fundamental diagram," Transportation Research Part B-Methodology, vol. 137, 2020.

[26] S. Sirmatel and N. Geroliminis, "Stabilization of city-scale road traffic networks via macroscopic fundamental diagrambased model predictive perimeter control," Control Engineering Practice, vol. 109, 2021.

[27] N. Zheng, K. Aboudolas, N. Geroliminis et al., "Investigation of a city-scale three-dimensional macroscopic fundamental diagram for Bi-modal urban traffic," in Proceedings of the 2013 16th International IEEE Conference on Intelligent Transportation Systems, pp. 1029-1034, Hague, The Netherlands, October 2013.

[28] N. Geroliminis, N. Zheng, and K. Ampountolas, "A threedimensional macroscopic fundamental diagram for mixed bimodal urban networks," Transportation Research Part C: Emerging Technologies, vol. 42, pp. 168-181, 2014.

[29] J. Ortigosa, M. Menendez, N. Zheng et al., "Analysis of the 3D-vMFDs of the urban networks of Zurich and San Francisco," in Proceedings of the 2015 IEEE 18th International Conference on Intelligent Transportation Systems, Spain, September 2015.

[30] T. Dantsuji, D. Fukuda, N. Zheng et al., "A macroscopic approach for optimizing road space allocation of bus lanes in multimodal urban networks through simulation analysis: an application to the Tokyo CBD network," in Proceedings of the 2017 IEEE 20th International Conference on Intelligent Transportation Systems, Yokohama, Japan, October 2017.

[31] F. Zhang, N. Zheng, H. Yang, and N. Geroliminis, "A systematic analysis of multimodal transport systems with road space distribution and responsive bus service," Transportation Research Part C: Emerging Technologies, vol. 96, pp. 208-230, 2018.

[32] I. Dakic, L. Ambühl, O. Schümperlin, and M. Menendez, "On the modeling of passenger mobility for stochastic bi-modal urban corridors," Transportation Research Part C: Emerging Technologies, vol. 113, pp. 146-163, 2020.

[33] M. Yingying, W. Chen, and J. Zehao, "Sensitivity analysis of MFD of ring radial road network on signal cycle," Journal of Transportation Systems Engineering and Information Technology, vol. 19, pp. 78-85+93, 2019. 